\title{
A facile way to produce epoxy nanocomposites having excellent thermal conductivity with low contents of reduced graphene oxide
}

\author{
Ganiu B. Olowojoba ${ }^{1, *}$, Sotirios Kopsidas ${ }^{1}$, Salvador Eslava ${ }^{2,3}$, Eduardo S. Gutierrez ${ }^{2}$, \\ Anthony J. Kinloch ${ }^{1}$, Cecilia Mattevi ${ }^{2}$, Victoria G. Rocha ${ }^{2,4}$, and Ambrose C. Taylor ${ }^{1}$ \\ ${ }^{1}$ Mechanics of Materials Division, Department of Mechanical Engineering, Imperial College London, London SW7 2AZ, UK \\ ${ }^{2}$ Centre for Advanced Structural Ceramics, Department of Materials, Imperial College London, London SW7 2AZ, UK \\ ${ }^{3}$ Department of Chemical Engineering, University of Bath, Bath BA2 7AY, UK \\ ${ }^{4}$ School of Engineering, Cardiff University, Cardiff CF24 $3 A A$, UK
}

Received: 15 December 2016

Accepted: 2 March 2017

Published online:

13 March 2017

(C) The Author(s) 2017. This article is published with open access at Springerlink.com

\begin{abstract}
A well-dispersed phase of exfoliated graphene oxide (GO) nanosheets was initially prepared in water. This was concentrated by centrifugation and was mixed with a liquid epoxy resin. The remaining water was removed by evaporation, leaving a GO dispersion in epoxy resin. A stoichiometric amount of an anhydride curing agent was added to this epoxy-resin mixture containing the GO nanosheets, which was then cured at $90^{\circ} \mathrm{C}$ for $1 \mathrm{~h}$ followed by $160{ }^{\circ} \mathrm{C}$ for $2 \mathrm{~h}$. A second thermal treatment step of $200{ }^{\circ} \mathrm{C}$ for $30 \mathrm{~min}$ was then undertaken to reduce further the GO in situ in the epoxy nanocomposite. An examination of the morphology of such nanocomposites containing reduced graphene oxide (rGO) revealed that a very good dispersion of rGO was achieved throughout the epoxy polymer. Various thermal and mechanical properties of the epoxy nanocomposites were measured, and the most noteworthy finding was a remarkable increase in the thermal conductivity when relatively very low contents of rGO were present. For example, a value of $0.25 \mathrm{~W} / \mathrm{mK}$ was measured at $30{ }^{\circ} \mathrm{C}$ for the nanocomposite with merely 0.06 weight percentage (wt $\%$ ) of rGO present, which represents an increase of $\sim 40 \%$ compared with that of the unmodified epoxy polymer. This value represents one of the largest increases in the thermal conductivity per wt $\%$ of added $\mathrm{rGO}$ yet reported. These observations have been attributed to the excellent dispersion of rGO achieved in these nanocomposites made via this facile production method. The present results show that it is now possible to tune the properties of an epoxy polymer with a simple and viable method of GO addition.
\end{abstract}

Address correspondence to E-mail: g.olowojoba@imperial.ac.uk 


\section{Introduction}

The starting material for the production of graphenebased polymer nanocomposites is frequently graphene oxide (GO), which is usually prepared either via an electrochemical method [1] or a chemical oxidation of graphite [2]. The latter method is often referred to as the Hummers method, or a variant of this process termed the modified Hummers method $[3,4]$. These very popular preparation routes lead to an aqueous dispersion of GO, with the GO containing many oxygen-containing functional groups (OCFGs) such as carboxyl, epoxide, carbonyl and hydroxyl groups. The attachment of the OCFGs during the chemical oxidation changes the hybridization of the carbon atoms in the graphitic lattice from $\mathrm{sp}^{2}$ to $\mathrm{sp}^{3}$. This disrupts electron and phonon transport, leading to poor electrical and thermal properties, respectively, of the GO so prepared, although it has the potential to increase the ease of dispersion of the GO when used as a filler in the production of polymer nanocomposites. The application of the graphenebased polymer nanocomposites requires that the OCFGs are reduced, to give reduced graphene oxide (rGO), in order to partially or fully obtain the excellent properties of graphene. Various reduction strategies for the GO are well known and have been reported in the literature [5-7]. For example, before the polymer nanocomposite is produced, the GO in the aqueous dispersion may be chemically reduced, using hazardous reagents such as hydrazine. Alternatively, the GO when dispersed in particulate form in the aqueous medium may then be obtained in a solid form, via filtration or freeze-drying, before it is reduced and then dispersed in the chosen matrix by sonication or shear mixing, or a combination thereof $[8,9]$. However, more recently, in situ reduction of the GO in the nanocomposite has gained in popularity [10-14]. This has arisen for several reasons. Firstly, if a good dispersion state of the GO exists in the aqueous medium it may be largely preserved, thereby circumventing the re-agglomeration which usually accompanies the reduction of the GO to rGO via the above-mentioned routes. Secondly, the in situ reduction route eliminates the extra processing step needed to reduce the GO prior to incorporation in a matrix. Thirdly, obtaining a well-dispersed phase of solid rGO in the polymeric matrix via sonication or shear mixing is far from easy. Therefore, the starting point of an aqueous dispersion of GO which is, as the final step, reduced to rGO in situ in the polymeric matrix offers a facile method for the production of such materials based upon an epoxy polymeric matrix.

Considering the work reported in the literature relevant to such a facile production method, Yang et al. [10] investigated GO/epoxy polymer nanocomposites produced by transferring the graphene oxide from an aqueous suspension into the epoxy resin via a two-phase extraction and then curing the well-dispersed GO/epoxy resin suspension. They reported significant improvements in the toughness and compressive failure strength (i.e. of 1185 and $48 \%$, respectively) of the epoxy nanocomposites containing as little as $0.038 \mathrm{wt} \%$ of GO. However, the relatively low reduction temperature of $150{ }^{\circ} \mathrm{C}$ that they adopted is likely to have left significant quantities of OCFGs on the GO and hence give a poor thermal conductivity after this relatively limited thermal treatment for the epoxy polymer nanocomposite. Peng et al. [15] adopted a similar approach to produce $\mathrm{rGO} /$ epoxy polymer nanocomposites with an excellent dispersion of rGO nanosheets. The reduction of the GO was carried out when the GO was suspended in the epoxy resin (triglycidyl paraaminophenol) at $200{ }^{\circ} \mathrm{C}$ for $5 \mathrm{~min}$, before curing the epoxy resin by adding 3,5-dimethylthio-2,4toluenediamine.

In this paper, we present a facile and effective route to prepare graphene-epoxy polymer nanocomposites with a remarkable increase in the thermal conductivity of the epoxy polymer at relatively very low contents of rGO. The ability of the OCFGs to impart a high degree of compatibility of the GO with epoxy resin is first exploited by mixing an aqueous dispersion of GO with the epoxy resin. Next, the remaining water is removed by evaporation, leaving a GO dispersion in the epoxy resin, which is cured with an anhydride curing agent. The final step is to reduce the GO in situ in the epoxy polymeric matrix at $200{ }^{\circ} \mathrm{C}$. The result is a good dispersion of rGO in the crosslinked epoxy polymer. The advantages of this facile production route, compared to previously reported routes, include: (a) the relatively high content of GO in the aqueous suspension used means that the processing time is considerably shorter; $(b)$ it does not involve the use of hazardous GO reducing agents such as hydrazine; (c) in situ reduction of the $\mathrm{GO}$ after curing of the epoxy polymer eliminates the extra processing step of reducing the GO prior to 
incorporation into the matrix; (d) the difficulties associated with dispersing powdery rGO into the epoxy resin are avoided; and (e) the relatively high temperature of $200{ }^{\circ} \mathrm{C}$ used to reduce the GO ensures an effective in situ reduction of the GO without degrading the chosen epoxy polymer. The thermal and mechanical properties of the epoxy nanocomposites so produced are reported in the present paper, together with the remarkable thermal conductivities at relatively very low contents of rGO.

\section{Experimental}

\section{Materials}

A standard diglycidyl ether of bis-phenol A (DGEBA) liquid epoxy resin (Araldite LY556; Huntsman, UK) having an epoxide equivalent weight (EEW) of $185 \mathrm{~g} / \mathrm{eq}$. was used. The curing agent was an accelerated methylhexahydrophthalic acid anhydride (Albidur HE600; Evonik, Germany) having an anhydride equivalent weight (AEW) of $170 \mathrm{~g} / \mathrm{eq}$.

\section{Synthesis of graphene oxide}

Graphene oxide was prepared via a modified Tour et al. [4] synthesis in a custom-built rig designed to employ up to $10 \mathrm{~L}$ of concentrated acid in two jacketed reactors with overhead stirrers. In a typical synthesis, a 10:1 mixture of concentrated acids (3 L $\mathrm{H}_{2} \mathrm{SO}_{4}: 0.3 \mathrm{~L} \mathrm{H}_{3} \mathrm{PO}_{4}$ ) was added to $24 \mathrm{~g}$ of natural graphite flakes (150-500 $\mu \mathrm{m}$; Sigma-Aldrich, UK) under vigorous stirring, followed by the addition of $144 \mathrm{~g}$ of $\mathrm{KMnO}_{4}$ (6 weight equivalent) in small portions. The reaction mixture was then kept at $50{ }^{\circ} \mathrm{C}$ under vigorous stirring for $18 \mathrm{~h}$. The mixture was cooled to room temperature, and the oxidation reactions were stopped by a drop-wise addition of $1.72 \mathrm{~L}$ of $2 \mathrm{wt} \%$ aqueous $\mathrm{H}_{2} \mathrm{O}_{2}$. The $\mathrm{GO}$ suspension was washed by repeated centrifugation and re-dispersion in distilled water, using a Sorvall LYNX 6000 Superspeed Centrifuge (Thermo Scientific, UK). This washing procedure was repeated until the $\mathrm{pH}$ of the supernatant matched that of the used distilled water, which typically occurred after 16 washing cycles. Typically two low-speed ( $<1000 \mathrm{rpm}$ ) centrifugation cycles were then performed to remove un-exfoliated graphite particles.

\section{Preparation of the epoxy polymer nanocomposites containing rGO}

A concentrated aqueous suspension of GO (13 mg/ $\mathrm{mL}$ ) was mixed with the epoxy resin by stirring first manually and then mechanically at $500 \mathrm{rpm}$ using a radial-flow impeller for $30 \mathrm{~min}$, as depicted in Fig. 1. The temperature was maintained at $60{ }^{\circ} \mathrm{C}$ to reduce the viscosity of the epoxy resin. Although aqueous GO suspensions are well known to form liquid crystalline structures at high contents [16], these are easily broken up by the mechanical agitation to allow a good dispersion of the aqueous GO suspension in the epoxy resin, owing to the compatibility between GO and epoxy. The resulting mixture was placed under vacuum at a gauge pressure of $-1000 \mathrm{mbar}$ and $60{ }^{\circ} \mathrm{C}$ for $2 \mathrm{~h}$ in order to evaporate the water and to remove any air bubbles. A stoichiometric amount of the anhydride curing agent was added to the GO/ epoxy mixture. The weight ratio of epoxy resin to anhydride curing agent was $185: 170$, as according to their equivalent weights. The resulting mixture was stirred at $500 \mathrm{rpm}$ for $15 \mathrm{~min}$ at a temperature of $60{ }^{\circ} \mathrm{C}$. The mixture was then degassed again at $-1000 \mathrm{mbar}$ and $60{ }^{\circ} \mathrm{C}$ for $15 \mathrm{~min}$ to remove air bubbles and any remaining water. The degassed mixture was poured into preheated rectangular steel moulds with internal dimensions of $150 \times 80 \times 3 \mathrm{~mm}^{3}$ and was cured at $90{ }^{\circ} \mathrm{C}$ for $1 \mathrm{~h}$ and then post-cured at $160{ }^{\circ} \mathrm{C}$ for $2 \mathrm{~h}$ to produce bulk nanocomposite plates. A further thermal treatment step of $200{ }^{\circ} \mathrm{C}$ for $30 \mathrm{~min}$ was then undertaken to ensure an effective in situ thermal reduction of the GO in the crosslinked epoxy polymer nanocomposite $[12,17]$. The contents of rGO prepared in the epoxy polymer nanocomposites were between 0.01 and 0.06 wt $\%$.

The unmodified epoxy polymer control was prepared in the same way as outlined above. However, in this case, the concentrated aqueous GO suspension was replaced with deionized water whose volume was equivalent to that of the aqueous rGO suspension used for the preparation of the nanocomposite with 0.06 wt $\%$ rGO (i.e. the highest rGO content used). 
Figure 1 Schematic of the nanocomposite production route. The GO is thermally reduced in situ after the curing of the epoxy polymer nanocomposite. The bottom left-hand photograph shows the cured nanocomposites containing increasing contents of rGO from left to right (0-0.06 wt \%).

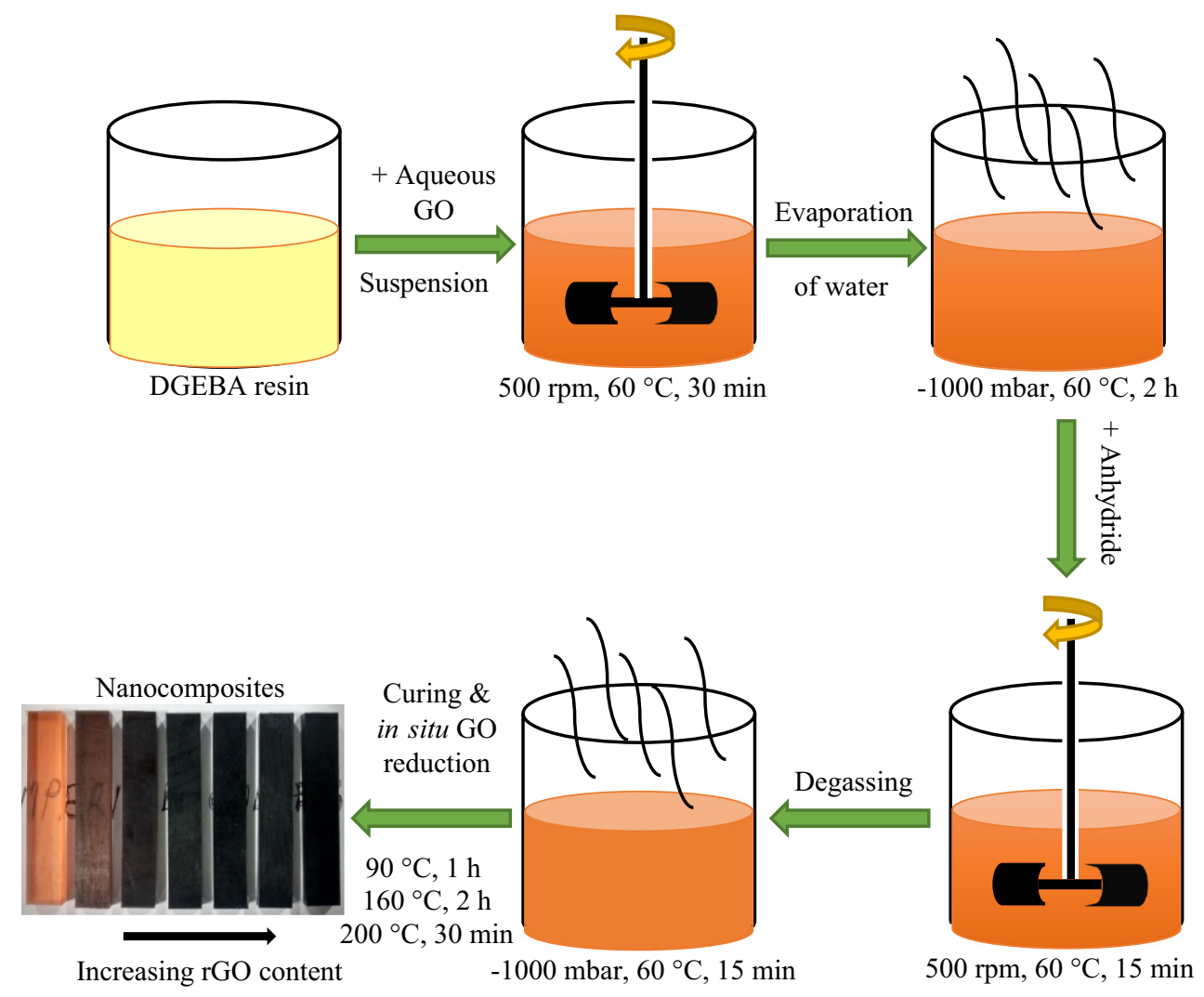

\section{Characterization}

\section{Microscopy studies}

Field-emission gun scanning electron microscopy (FEG-SEM) was used to image the dispersion of the rGO by examination of fractured samples of the nanocomposites. A Leo 1525 (Carl Zeiss, Germany) microscope was used, with an accelerating voltage of $5 \mathrm{kV}$. The samples were sputter-coated with a thin film of chromium prior to examination. Transmission electron microscopy (TEM) was carried out using a 2000FX microscope (JEOL, USA) employing an accelerating voltage of $200 \mathrm{kV}$. Thin slices (about $70 \mathrm{~nm}$ in thickness) were cut for TEM using a PowerTome XL ultramicrotome (RMC Products, UK). Atomic force microscopy (AFM) was carried out to determine the morphology of the GO using a MultiMode scanning probe microscope (Veeco, UK). The microscope was equipped with a NanoScope IV controller and an E scanner. The concentrated aqueous GO suspension $(13 \mathrm{mg} / \mathrm{mL})$ was diluted to $0.1 \mathrm{mg} / \mathrm{mL}$ using deionized water. The diluted GO suspension was then dip-coated onto a mica sheet (Agar Scientific, UK) and allowed to dry. Height and phase images were captured by AFM at a resolution of 512 pixels $\times 512$ pixels and a scan speed of $1 \mathrm{~Hz}$, using silicon probes in a tapping mode of operation.

\section{Infrared spectroscopy studies}

Fourier transform infrared spectroscopy (FTIR) was carried out on freeze-dried GO and thermallyreduced GO samples using a Spectrum 100 FTIR Spectrometer (PerkinElmer, UK). Aqueous GO suspensions, produced as outlined above, were freezedried using a Powerdry LL1500 freeze dryer (Thermo Scientific, UK) to obtain the dry GO powder used for FTIR and TGA. The freeze-dried GO was thermally reduced according to the cure plus reduction schedule adopted for the production of the nanocomposites (i.e. $90{ }^{\circ} \mathrm{C}$ for $1 \mathrm{~h}, 160{ }^{\circ} \mathrm{C}$ for $2 \mathrm{~h}$ and $200{ }^{\circ} \mathrm{C}$ for $30 \mathrm{~min})$.

\section{Mechanical and thermo-mechanical studies}

Uniaxial tensile tests were conducted at room temperature using a universal testing machine (5584; Instron, UK). Dumbbell specimens having a gauge length of $25 \mathrm{~mm}$ were machined from 
$75 \times 13.5 \times 3 \mathrm{~mm}^{3}$ pieces cut from the bulk nanocomposite plates. A displacement rate of $1 \mathrm{~mm} /$ min was used, and the strain was measured using a clip-on extensometer (2620-601; Instron, UK). Tensile modulus values were calculated between strains of 0.05 and $0.25 \%$. The tensile properties were averaged from the results obtained from a minimum of five specimens.

The thermo-mechanical properties of the nanocomposites were determined by dynamic mechanical thermal analysis (DMTA) using a Q800 analyser (TA Instruments, UK). Rectangular samples with dimensions of $60 \times 10 \times 3 \mathrm{~mm}^{3}$ were cut from the bulk nanocomposite plates. The samples were subjected to a temperature sweep from 30 to $200{ }^{\circ} \mathrm{C}$ at a heating rate of $2{ }^{\circ} \mathrm{C} / \mathrm{min}$ in dual-cantilever mode at a frequency of $1 \mathrm{~Hz}$ using an oscillation strain of $0.05 \%$. The glass transition temperature, $T_{\mathrm{g}}$, of each sample was taken at the maximum of the $\tan \delta$ curve. The number average molecular weight between crosslinks, $M_{\text {nc }}$ was calculated from:

$M_{\mathrm{nc}}=\frac{q \rho R T}{E_{\mathrm{r}}}$

where $q$ is the front factor, $\rho$ is the density of the epoxy determined at room temperature $\left(1.2 \mathrm{~g} / \mathrm{cm}^{3}\right.$ [18]), $R$ is the universal gas constant $(8.314 \mathrm{~J} / \mathrm{kg} \mathrm{K})$, $T$ is the temperature and $E_{\mathrm{r}}$ is the rubbery storage modulus determined at a temperature of $T=453 \mathrm{~K}$ $\left(180^{\circ} \mathrm{C}\right)$. Since the density of the epoxy was determined at room temperature, Pearson and Yee [18] suggest a front factor, $q$, of 0.725 .

\section{$X$-ray studies}

X-ray photoelectron spectroscopy (XPS) analysis of the GO and rGO was performed using a Theta Probe spectrometer (ThermoFisher Scientific, UK), operated at a base pressure of $1 \times 10^{-9} \mathrm{mbar}$. The spectra were acquired using a MXR1 monochromated Al Ka X-ray source $(h v=1486.6 \mathrm{eV})$. An X-ray spot of $\sim 400 \mu \mathrm{m}$ radius was employed. High-resolution, core-level C1s spectra were acquired using a pass energy of $20 \mathrm{eV}$. The GO sample spectra were charge-referenced against the $\mathrm{C} 1 \mathrm{~s}$ peak at $284.4 \mathrm{eV}$ to correct for charging effects during acquisition. Quantitative surface chemical analyses were calculated from the high-resolution, core-level spectra following the removal of a nonlinear (Shirley) background. The manufacturer's Avantage software was used which incorporates the appropriate sensitivity factors and corrects for the electron energy analyser transmission function.

X-ray diffraction (XRD) patterns of the samples were acquired using an X'Pert PRO diffractometer (PANalytical, UK). The diffractometer was equipped with a $\mathrm{Cu} \mathrm{K} \alpha$ radiation source. A generator voltage of $40 \mathrm{kV}$ and tube current of $40 \mathrm{~mA}$ were used for all measurements over a $2 \theta$ range of $5^{\circ}-60^{\circ}$, using a step size of $0.02^{\circ}$ and $20 \mathrm{~s}$ per step.

\section{Thermal studies}

Thermogravimetric analysis (TGA) was carried out on the nanocomposites and freeze-dried GO samples using a TGA/DSC 1 (Mettler Toledo, UK). The samples were analysed using a heating rate of $10{ }^{\circ} \mathrm{C} / \mathrm{min}$ over a temperature range of $30-800{ }^{\circ} \mathrm{C}$ in either an air or a nitrogen atmosphere.

The thermal conductivities of the nanocomposites were determined by the laser flash technique using an LFA-427 Nanoflash (NETZSCH, Germany). The samples were coated with a thin layer of graphite prior to testing to increase absorption and transmission of infrared radiation energy. Three shots each were made on $10 \mathrm{~mm} \times 10 \mathrm{~mm}$ samples, $2 \mathrm{~mm}$ thick, over a temperature range of $30-60$ at $10^{\circ} \mathrm{C}$ intervals. A laser voltage of $450 \mathrm{~V}$ and a pulse width of $0.8 \mathrm{~ms}$ were used. The thermal diffusivity of each sample was measured and converted to the thermal conductivity using the specific heat capacity estimated from that of a graphite control sample whose thermal diffusivity had been determined using the same set of conditions.

\section{Results and discussion}

\section{Dispersion quality of GO in the aqueous suspension}

Preparation of the aqueous suspension of GO via the modified Hummers method leads to intercalation of OCFGs between the GO sheets formed during the oxidation of the graphite. This leads to an increase in the interlayer separation and hence a weakening of the attractive forces between the GO sheets. Moreover, the presence of the OCFGs imparts a high degree of compatibility of the GO with water and subsequently with the epoxy resin. These factors 
make it relatively easy to exfoliate and disperse the GO, with a minimum energy input, in the aqueous suspension. To illustrate this, a concentrated aqueous GO suspension of a content of $13 \mathrm{mg} / \mathrm{mL}$ was diluted to $0.1 \mathrm{mg} / \mathrm{mL}$ by adding deionized water, and the resulting suspension was vigorously shaken. The GO suspension was then dip-coated onto a mica sheet, dried and examined using AFM. Figure 2a shows the height image obtained, and Fig. 2b shows the height profile measured along the line indicated in Fig. 2a. A mean height of $\sim 0.9 \mathrm{~nm}$ was measured for the GO deposited on the mica substrate. Now, a GO sheet has been reported as having a thickness in the range of $0.5-1.1 \mathrm{~nm}$ [5]. Thus, the measured thickness of the deposited GO indicates the occurrence of isolated individual GO sheets in the dilute aqueous suspension. This clearly demonstrates the ease with which GO can be readily exfoliated and dispersed with a minimal energy input.
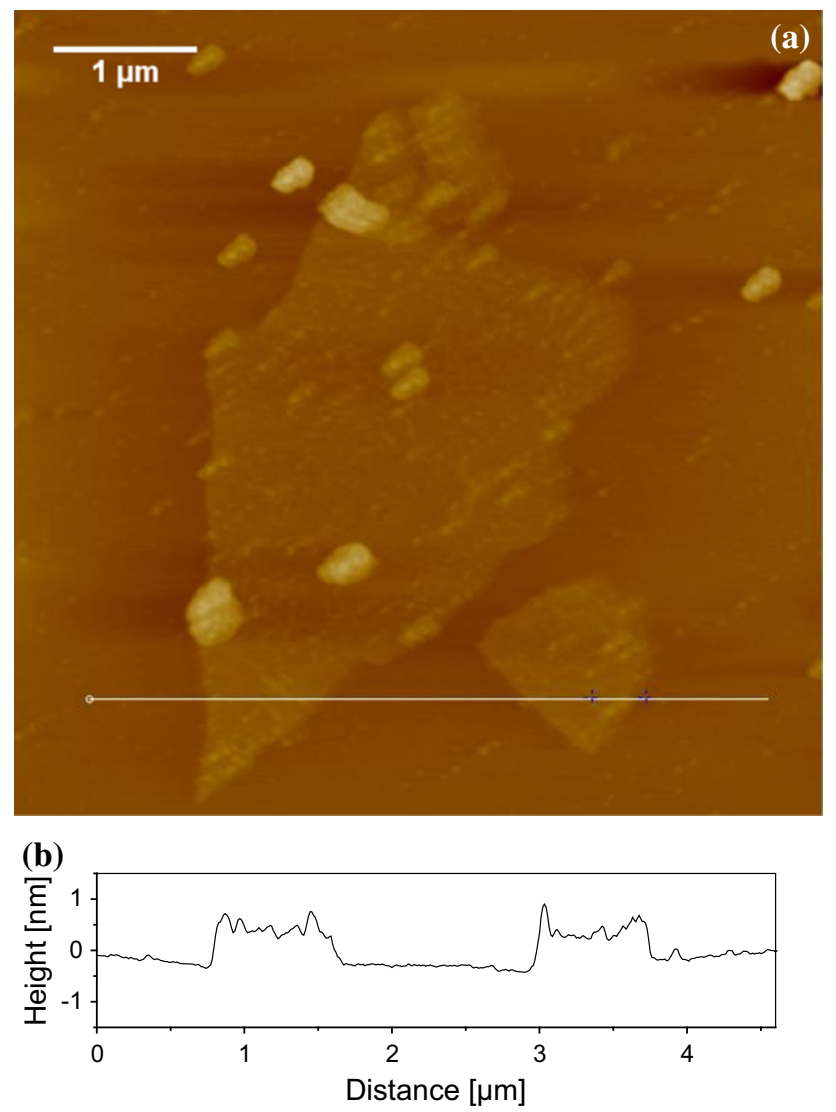

Figure 2 a AFM height image of GO sheets dip-coated onto a mica sheet from an aqueous solution of $0.1 \mathrm{mg} / \mathrm{mL}$ and $\mathbf{b}$ height profile along line shown in (a).

\section{Selection of temperature for the reduction of graphene oxide}

Reduction of the GO was carried out in situ in the cured epoxy polymer; however, to establish suitable reduction conditions, it was necessary to carry out preliminary tests on freeze-dried (powdery) GO. TGA was performed on this powdery GO in a nitrogen atmosphere, and the resulting thermogram is shown in Fig. 3. The $15 \%$ mass loss at $100{ }^{\circ} \mathrm{C}$ is due to the removal of adsorbed hydroxyls and the byproducts of low-temperature reduction of the GO, e.g. $\mathrm{O}_{2}$ and $\mathrm{H}_{2} \mathrm{O}$ [19]. A further $30 \%$ mass loss at $200{ }^{\circ} \mathrm{C}$ can be attributed to the thermal decomposition of OCFGs on the surface of the GO. It has previously been shown that most of the OCFGs attached to the basal aromatic plane of a GO sheet (hydroxyl and epoxide groups) can be removed by thermal treatment at $200{ }^{\circ} \mathrm{C}$ [20], and this largely restores the thermal, electrical and optical properties to those of graphene. Tang et al. [12] demonstrated that simply heating GO to $200{ }^{\circ} \mathrm{C}$ removes most of the OCFGs on the aromatic plane of GO and that holding the GO at this temperature for longer only leads to a marginal further increase in the weight of OCFGs lost. However, the OCFGs which are attached to the edges of the GO sheets are more thermally stable and will only decompose at temperatures higher than $200{ }^{\circ} \mathrm{C}$ [5], as seen from the gradual mass loss in the temperature range from 300 to $800{ }^{\circ} \mathrm{C}$, see Fig. 3. Although it would be useful to reduce the GO at temperatures higher than $200{ }^{\circ} \mathrm{C}$, degradation of the epoxy polymer will occur. Avoiding such degradation limits the reduction temperature that can be used.

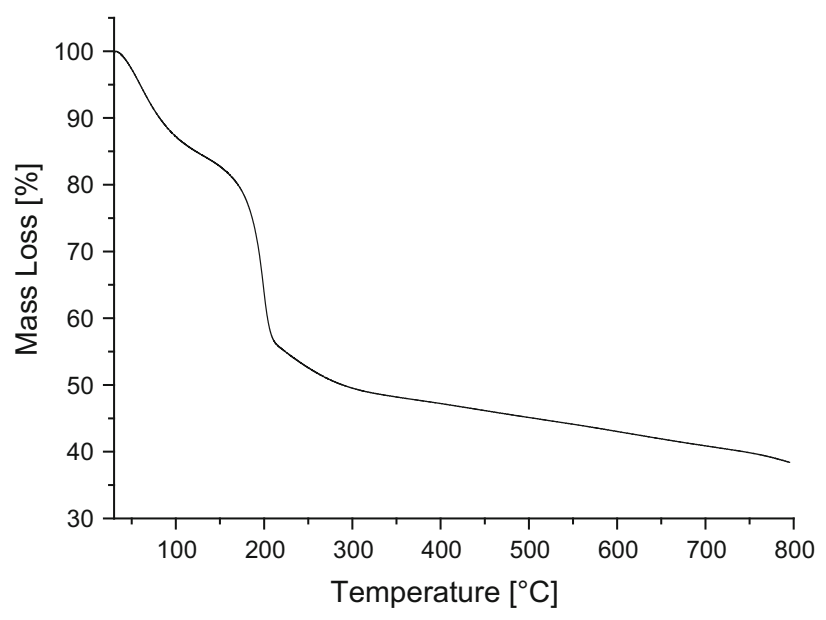

Figure 3 Thermogram of GO in a nitrogen atmosphere. 


\section{Effect of thermal treatment time at $200{ }^{\circ} \mathrm{C}$ on the reduction of $\mathrm{GO}$}

Once the reduction temperature of $200{ }^{\circ} \mathrm{C}$ was selected, the effect of the thermal treatment time on GO was investigated to allow a suitable reduction time to be chosen. Freeze-dried (powdery) GO samples were reduced at $200{ }^{\circ} \mathrm{C}$ for different lengths of time ranging from $10 \mathrm{~min}$ to $6 \mathrm{~h}$, and Fig. 4a shows the resulting $X$-ray diffraction patterns of the rGO samples. The measured peak positions and interlayer spacings are shown in Table 1 . All the rGO samples showed (002) diffraction peaks at $\sim 24.4^{\circ}$, corresponding to an interlayer spacing of $\sim 0.36 \mathrm{~nm}$. No significant change was observed in the peak positions and interlayer spacings of the rGO samples, indicating that the amount of OCFGs removed by thermal dissociation does not correlate strongly with the reduction time of the GO.

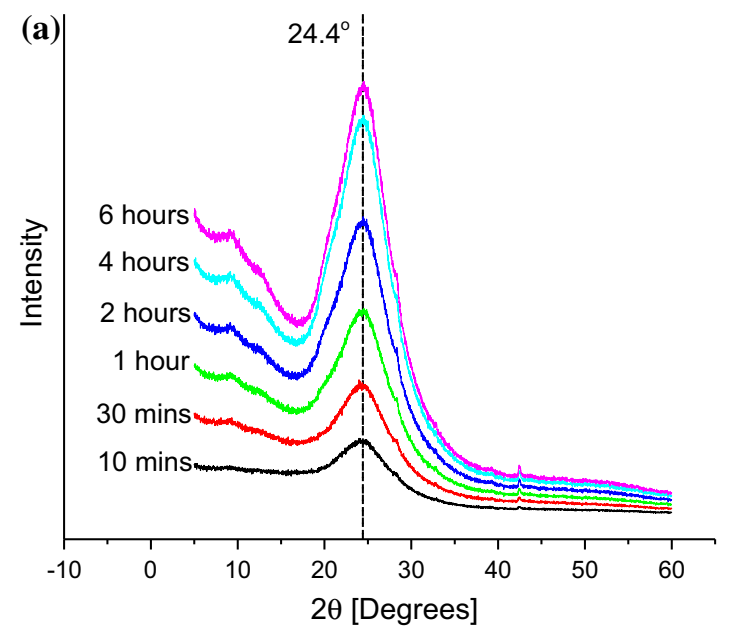

The thermograms obtained in air for the rGO samples are presented in Fig. $4 \mathrm{~b}$. The values of the mass loss over the temperature range of $250-450{ }^{\circ} \mathrm{C}$ are shown in Table 1, and this reduction can be attributed to the loss of OCFGs which are attached to the edges of the GO sheets. The mass loss in the temperature range of $450-650{ }^{\circ} \mathrm{C}$ can be attributed to the thermal oxidation of the graphene material. Table 1 also shows that the mass loss in the temperature range of $250-450{ }^{\circ} \mathrm{C}$ is largely insensitive to the GO thermal treatment time. The XPS spectra of the rGO samples are shown in Fig. 5a, while the corresponding high-resolution C1s spectra are shown in Fig. 5b. Peak deconvolution allows the quantification of the relative amounts of the different moieties bonded to the carbon atoms, as shown in Table 1 .

There is little variation in the relative amounts of the carbon atoms bonded to other carbon atoms or to OCFGs as the thermal treatment time is

Figure 4 Effect of treatment time on the reduction of GO as studied by $\mathbf{a}$ XRD and $\mathbf{b}$ TGA.

Table $1 \mathrm{XRD}$, TGA and XPS data for rGO samples after thermal treatment at $200{ }^{\circ} \mathrm{C}$ for different periods of time

\begin{tabular}{|c|c|c|c|c|c|c|c|}
\hline \multirow{2}{*}{$\begin{array}{l}\text { GO thermal treatment } \\
\text { time }(\mathrm{h})\end{array}$} & \multicolumn{2}{|c|}{$\begin{array}{l}\text { XRD peak properties and } \\
\text { interlayer spacing }\end{array}$} & \multirow{2}{*}{$\begin{array}{l}\text { TGA mass loss, } \\
250-450{ }^{\circ} \mathrm{C}(\%)\end{array}$} & \multicolumn{4}{|c|}{ XPS abundance of functional groups (\%) } \\
\hline & $\begin{array}{l}\text { Position } \\
\text { (degrees) }\end{array}$ & $\begin{array}{l}\text { d-spacing } \\
(\mathrm{nm})\end{array}$ & & $\begin{array}{l}\mathrm{C}-\mathrm{C} \\
(284.7 \mathrm{eV})\end{array}$ & $\begin{array}{l}\mathrm{C}-\mathrm{OH} \\
(286.3 \mathrm{eV})\end{array}$ & $\begin{array}{l}\mathrm{C}=\mathrm{O} \\
(288.7 \mathrm{eV})\end{array}$ & $\begin{array}{l}\mathrm{HO}-\mathrm{C}=\mathrm{O} \\
(289 \mathrm{eV})\end{array}$ \\
\hline 0.17 & 24.42 & 0.364 & 8.01 & 73.45 & 20.41 & 2.83 & 3.30 \\
\hline 0.5 & 24.47 & 0.364 & 8.50 & 69.97 & 21.48 & 3.51 & 5.04 \\
\hline 1 & 24.57 & 0.362 & 8.31 & 69.47 & 20.53 & 4.68 & 5.32 \\
\hline 2 & 24.46 & 0.364 & 8.93 & 72.64 & 17.40 & 3.44 & 6.53 \\
\hline 4 & 24.57 & 0.362 & 8.30 & 73.65 & 18.06 & 3.24 & 5.06 \\
\hline 6 & 25.03 & 0.356 & 9.20 & 72.79 & 19.95 & 2.85 & 4.41 \\
\hline
\end{tabular}



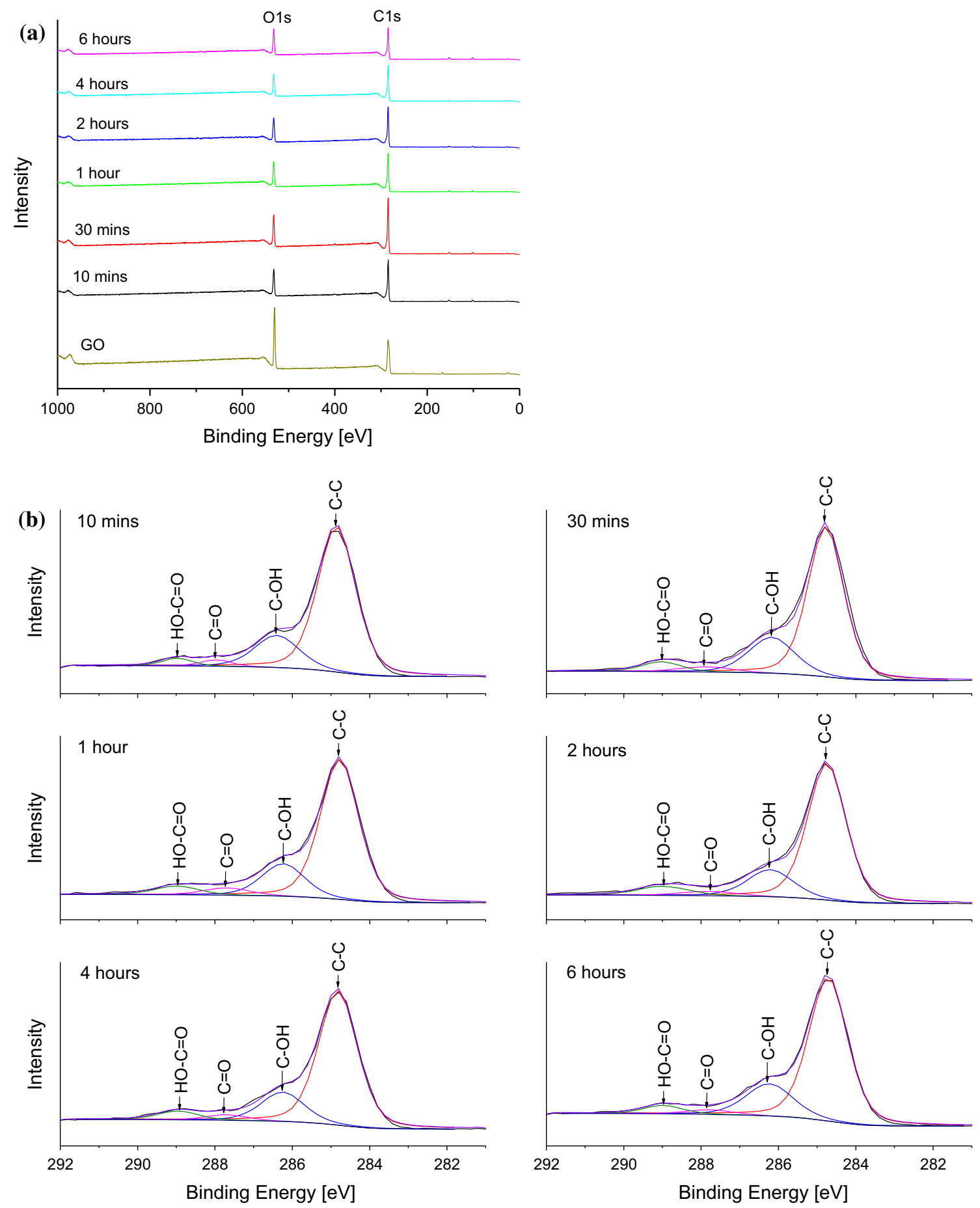

Figure 5 Effect of thermal treatment time on the reduction of GO as studied by XPS a survey spectra and $\mathbf{b}$ high-resolution C1s spectra.

increased. Although Jeong et al. [20] have previously indicated that the relative amount of OCFGs removed by thermal decomposition could be a function of the thermal treatment time, Tang et al.
[12] concluded that the GO thermal treatment time has no effect. The results shown indicate that the GO thermal treatment time has little or no effect on the amount of OCFGs removed at $200{ }^{\circ} \mathrm{C}$. 


\section{Effect of the chosen reduction schedule for GO}

Based on the results of the above experiments, to reduce the GO without degrading the epoxy polymer, the reduction of the GO in the cured epoxy polymer nanocomposite was carried out in situ using a thermal treatment schedule of $200{ }^{\circ} \mathrm{C}$ for $30 \mathrm{~min}$. Figure 6a compares the results of TGA in a nitrogen atmosphere of powdery GO before reduction with that of rGO reduced according to the complete cure and reduction schedule discussed above (i.e. $90{ }^{\circ} \mathrm{C}$ for $1 \mathrm{~h}, 160{ }^{\circ} \mathrm{C}$ for $2 \mathrm{~h}$ and $200^{\circ} \mathrm{C}$ for $30 \mathrm{~min}$ ). For the $\mathrm{rGO}$, the $7 \%$ mass loss at $100{ }^{\circ} \mathrm{C}$ may be attributed to the removal of adsorbed hydroxyls and the byproducts of low-temperature reduction of the GO,
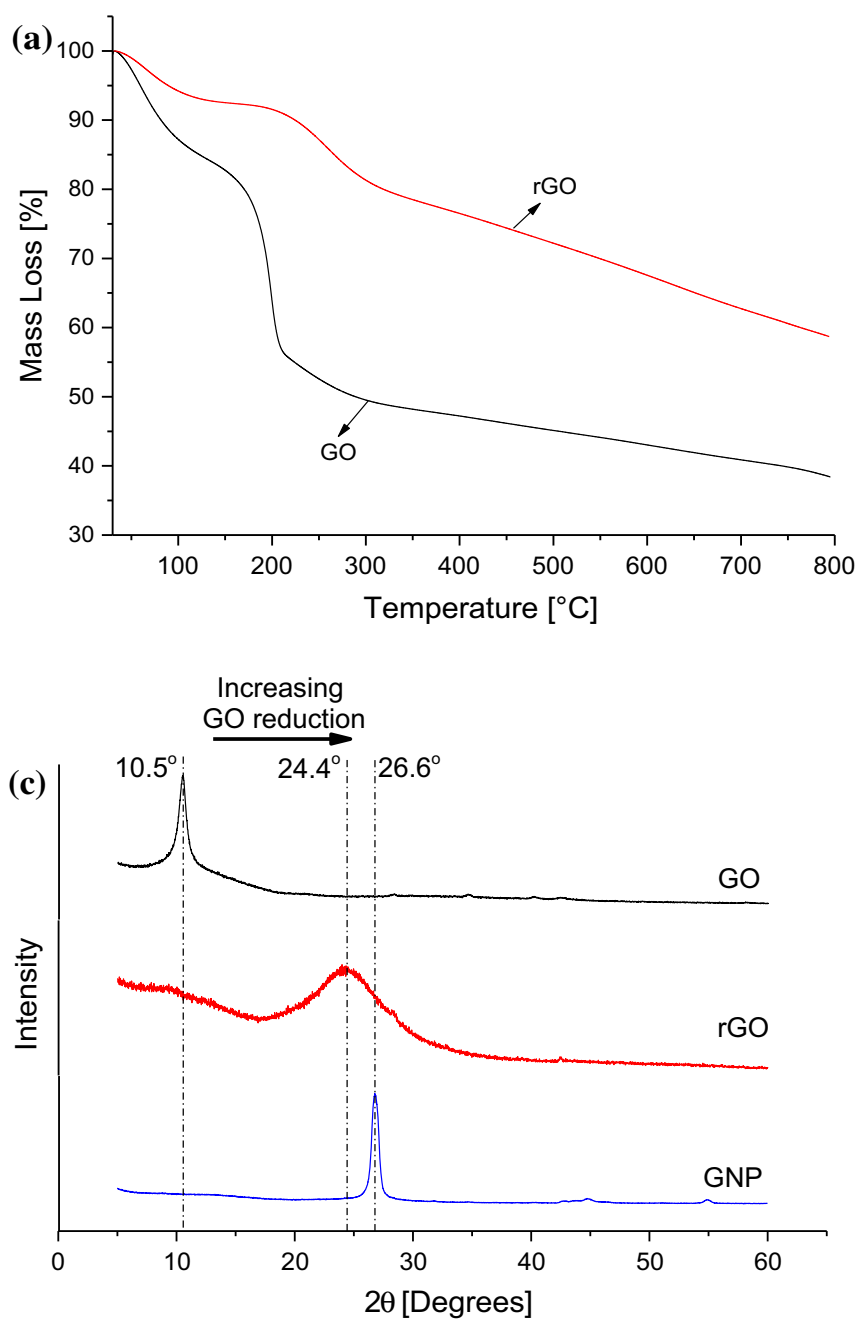

Figure 6 a Thermograms of $\mathrm{GO}$ and $\mathrm{rGO}$ in a nitrogen atmosphere, $\mathbf{b}$ FTIR spectra of GO and rGO, c XRD patterns of GO before and after reduction (the XRD pattern of a e.g. $\mathrm{O}_{2}$ and $\mathrm{H}_{2} \mathrm{O}$ [19], see Fig. 6a. Although the thermal treatment to form rGO removes most of the OCFGs on the aromatic plane of GO, which decompose at $200{ }^{\circ} \mathrm{C}$, the OCFGs on the edges of the GO sheets are more thermally stable. These decompose at higher temperatures [5], giving rise to the gradual mass loss between 300 and $800{ }^{\circ} \mathrm{C}$ in Fig. 6a.

Figure $6 \mathrm{~b}$ shows the FTIR spectra of the powdery $\mathrm{GO}$ and rGO. For GO, a broad peak corresponding to adsorbed hydroxyl groups from $\mathrm{C}-\mathrm{OH}, \mathrm{HO}-\mathrm{C}=\mathrm{O}$ and $\mathrm{H}-\mathrm{OH}$ can be seen between 3146 and $3377 \mathrm{~cm}^{-1}$. The peaks at 1734, 1636, 1621.5 and $1223.5 \mathrm{~cm}^{-1}$ correspond to $\mathrm{C}=\mathrm{O}, \mathrm{HO}-\mathrm{C}=\mathrm{O}, \mathrm{C}=\mathrm{C}$ and $\mathrm{C}-\mathrm{O}-\mathrm{C}$ stretching, respectively, while those at 1351 and $1047 \mathrm{~cm}^{-1}$ correspond to $\mathrm{C}-\mathrm{OH}$ stretching in the basal aromatic plane and at the edge of the GO,
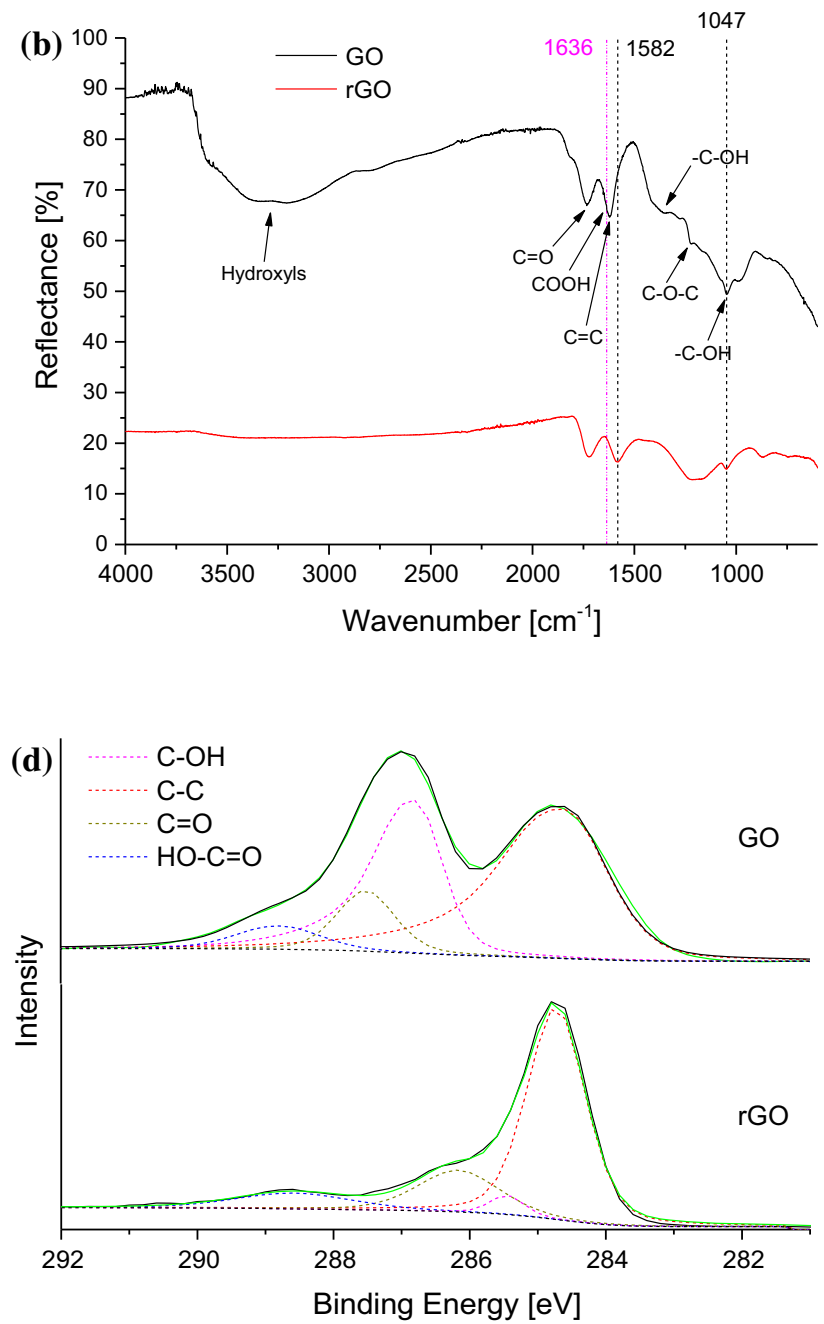

commercially-available GNP is also shown for comparison) and d XPS of GO before and after reduction, showing the decrease in the concentrations of OCFGs present after reduction. 


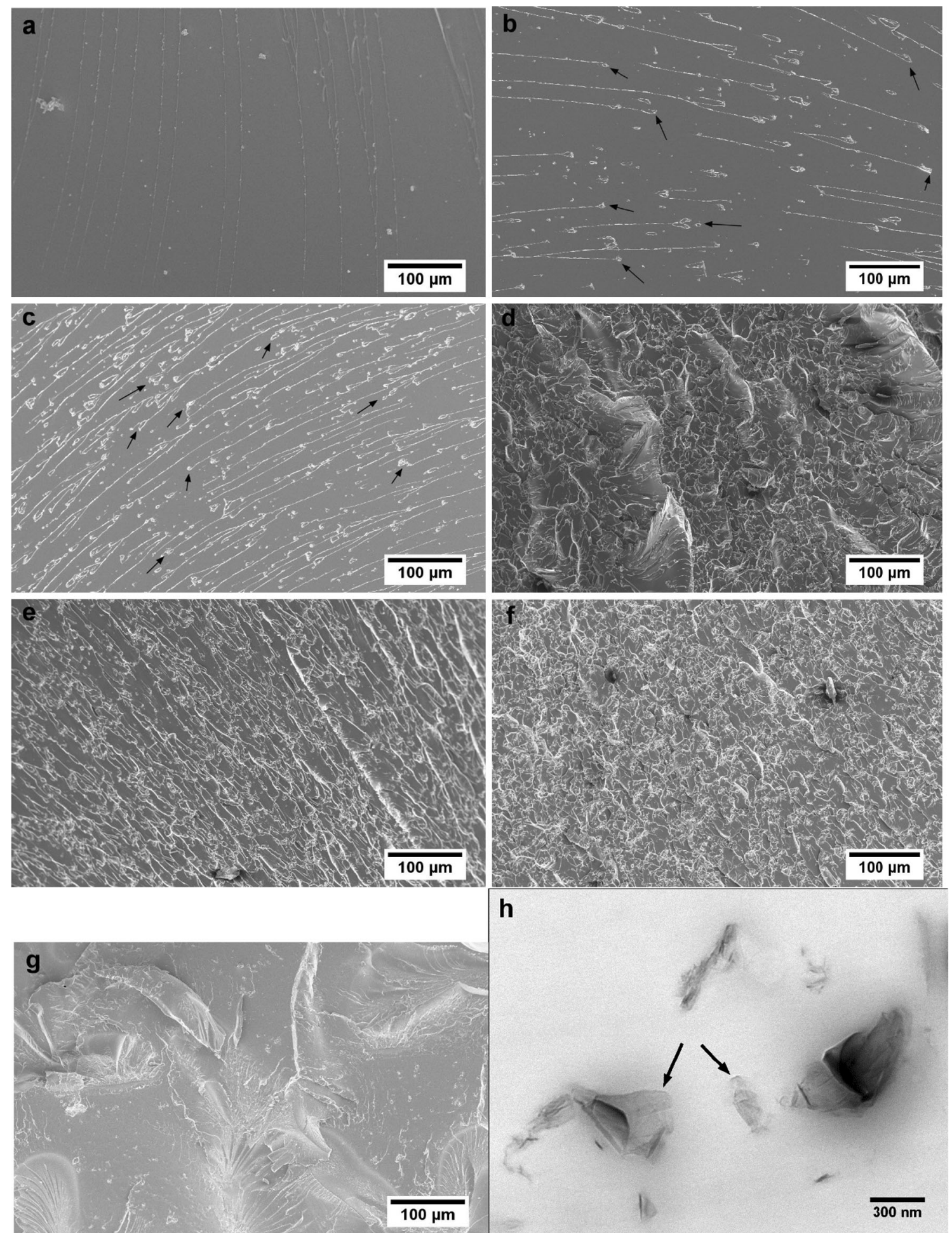

Figure 7 FEG-SEM of fractured surfaces of rGO/epoxy nanocomposites for a unmodified epoxy, b $0.01 \mathrm{wt} \%$, c $0.02 \mathrm{wt} \%$, d $0.03 \mathrm{wt} \%$, e $0.04 \mathrm{wt} \%$, f $0.05 \mathrm{wt} \%, \mathbf{g} 0.06 \mathrm{wt} \%$ of rGO and $\mathbf{h}$ TEM of $0.06 \mathrm{wt} \%$ nanocomposite showing isolated sheets of rGO.

respectively [5, 21-23]. After reduction, several changes can be observed in the infrared absorption spectrum. Firstly, the peak at $1636 \mathrm{~cm}^{-1}$ corresponding to $\mathrm{HO}-\mathrm{C}=\mathrm{O}$ disappears, while the $\mathrm{C}-\mathrm{OH}$ and $\mathrm{C}-\mathrm{O}-\mathrm{C}$ peaks originally at 1351 and $1223.5 \mathrm{~cm}^{-1}$, respectively, in GO are replaced by a broad peak of cyclic moieties in rGO. It can be seen, however, that the peak at $1047 \mathrm{~cm}^{-1}$ which corresponds to the 

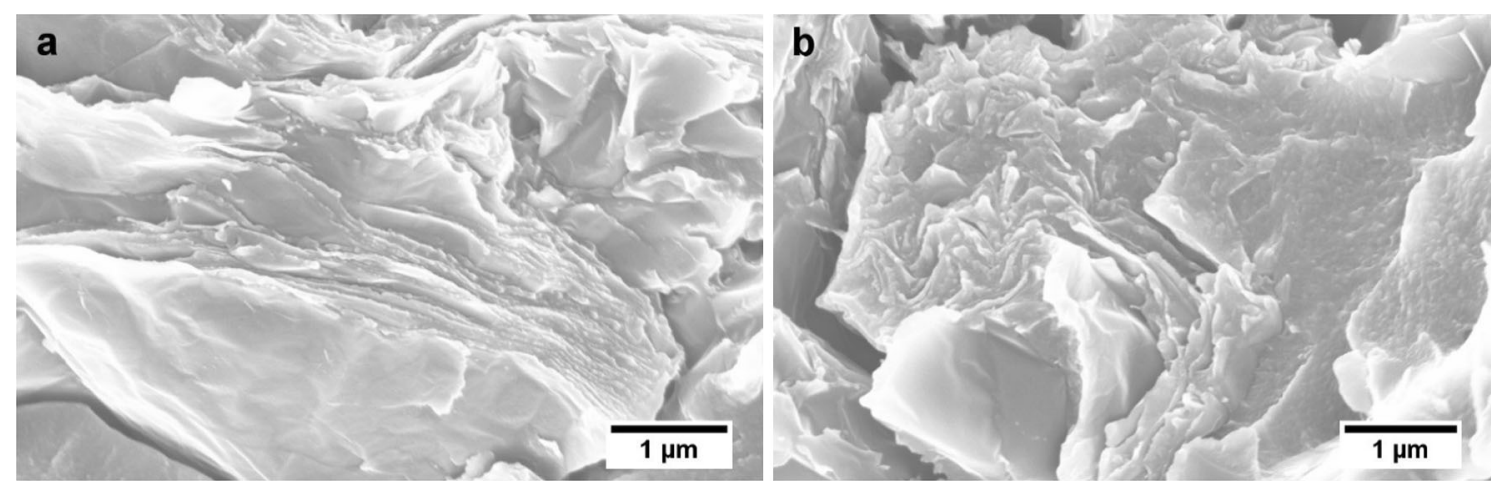

Figure 8 FEG-SEM images of 0.06 wt $\%$ rGO nanocomposite, showing self-assembly of rGO sheets.

stretching of $\mathrm{C}-\mathrm{OH}$ attached to the edge of the $\mathrm{GO}$ sheet is unchanged. It is believed that these hydroxyl groups which are attached to the edge of the GO sheet are very stable and will only dissociate at temperatures in excess of $650{ }^{\circ} \mathrm{C}$ [5]. Tang et al. [12] obtained similar results. In addition to the removal of OCFGs during reduction, it can be seen that the $\mathrm{C}=\mathrm{C}$ stretching peak is shifted from 1636 to $1582 \mathrm{~cm}^{-1}$. This is indicative of an increase in the recovery of $\mathrm{sp}^{2}$ hybridized carbon domains after the reduction of the GO.

The position of the (002) diffraction peak in XRD can usually be correlated to the size of the diffraction grating (in this case, the chemical heterogeneity of the GO largely determines the interlayer spacing). The reduction in chemical heterogeneity as the GO is reduced can be seen very clearly by the position of the diffraction peaks in the XRD patterns of GO and rGO shown in Fig. 6c. The XRD pattern of commercially-available thermally-reduced graphene nanoplatelets (GNPs) is shown for comparison. It can be seen that the (002) diffraction peak at $2 \theta=26.6^{\circ}$ in GNP is shifted to $2 \theta=10.5^{\circ}$ in GO after chemical oxidation. However, the (002) peak is partially restored to $2 \theta=24.4^{\circ}$ in rGO as many of the OCFGs are removed during thermal reduction. The corresponding interlayer spacing, as measured, decreases from $0.84 \mathrm{~nm}$ in GO to $0.36 \mathrm{~nm}$ in $\mathrm{rGO}$ as the OCFGs intercalated between individual sheets are removed [24-26]. By comparison, the measured interlayer spacing of pristine GNPs is $0.34 \mathrm{~nm}$. The GO reduction process often leads to increased dislocations and imperfections in the graphitic lattice as some carbon atoms are lost in the form of $\mathrm{CO}_{2}$ or $\mathrm{CO}$ during reduction [5]. These lattice imperfections, as well as stacking faults generated in the restacking of graphitic sheets during reduction, may be contributing factors to the broadening of the (002) peak in rGO as observed in Fig. 6c [26].

Figure $6 \mathrm{~d}$ shows the high-resolution C1s XPS spectra of GO and rGO, as well as the associated deconvoluted peaks which quantitatively represent the bonds between carbon atoms on the GO and other moieties. The relative abundance of the $\mathrm{C}=\mathrm{C}$ bonds increased from $50.61 \%$ for GO to $69.97 \%$ for rGO. A decrease in the OCFGs bonded to carbon atoms was, however, observed; the $\mathrm{C}-\mathrm{OH}$ groups decreased from $33.49 \%$ in $\mathrm{GO}$ to $21.48 \%$ in $\mathrm{rGO}$, the $\mathrm{C}=\mathrm{O}$ groups decreased from $10.19 \%$ in $\mathrm{GO}$ to $3.51 \%$ in $\mathrm{rGO}$, and the $\mathrm{HO}-\mathrm{C}=\mathrm{O}$ groups decreased from $5.71 \%$ of $\mathrm{GO}$ to $5.04 \%$ in rGO. A significant reduction in the concentrations of the OCFGs bonded to carbon atoms can thus be observed upon reduction of GO, in accordance with the TGA, FTIR and XRD results previously discussed. These results clearly demonstrate the effectiveness of the GO reduction strategy adopted in the present work for the preparation of the rGO/epoxy nanocomposites.

\section{Dispersion of rGO in the nanocomposites}

For the rGO/epoxy polymer nanocomposites, where the GO is reduced in situ, it is instructive to first consider how the rGO is dispersed in the polymer matrix. The size and dispersion of the rGO sheets can be imaged using FEG-SEM on fractured samples. For convenience, the fracture surfaces of samples broken during tensile tests were used. (Note that the results of the tensile tests are discussed separately below). The unmodified epoxy is a homogeneous polymer as expected, see Fig. 7a. Note that the white lines in Fig. 7a are river lines caused by the brittle fracture of the polymer [27], and for the purposes of considering 

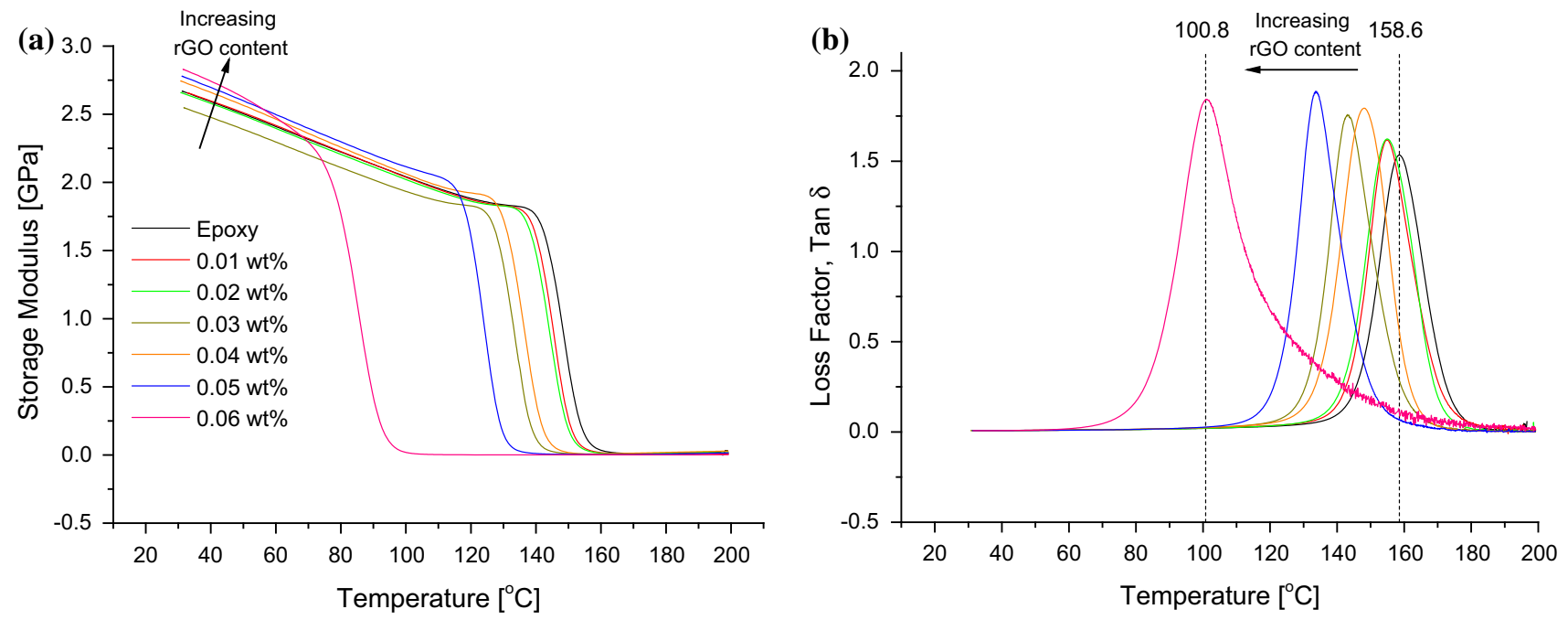

Figure 9 Thermo-mechanical properties of the rGO/epoxy polymer nanocomposites with varying rGO content showing a storage modulus and $\mathbf{b} \tan \delta$.

Table 2 Thermo-mechanical properties of rGO/epoxy nanocomposites as obtained by DMTA

\begin{tabular}{|c|c|c|c|c|c|}
\hline $\begin{array}{l}\text { rGO } \\
\text { content } \\
\left(\mathrm{wt}^{2} \%\right)\end{array}$ & $\begin{array}{l}\text { Storage modulus }\left(E_{\mathrm{g}}{ }^{\prime}\right) \\
\text { at } 40{ }^{\circ} \mathrm{C}(\mathrm{GPa})\end{array}$ & $\begin{array}{l}\text { Storage modulus }\left(E_{\mathrm{r}}^{\prime}\right) \\
\text { at } 180{ }^{\circ} \mathrm{C}(\mathrm{MPa})\end{array}$ & $\begin{array}{l}\text { Glass transition } \\
\text { temperature }\left(T_{\mathrm{g}}\right)\left({ }^{\circ} \mathrm{C}\right)\end{array}$ & $\begin{array}{l}\text { Height of } \\
\tan \delta \text { peak }\end{array}$ & $\begin{array}{l}\text { Molecular weight between } \\
\text { crosslinks }\left(M_{\mathrm{nc}}\right)\left(\mathrm{gmol}^{-1}\right)\end{array}$ \\
\hline 0.00 & 2.59 & 11.5 & 158.6 & 1.53 & 285 \\
\hline 0.01 & 2.60 & 10.1 & 154.9 & 1.61 & 324 \\
\hline 0.02 & 2.58 & 9.8 & 154.2 & 1.62 & 334 \\
\hline 0.03 & 2.48 & 7.6 & 143.0 & 1.76 & 431 \\
\hline 0.04 & 2.66 & 8.2 & 148.1 & 1.79 & 399 \\
\hline 0.05 & 2.70 & 3.7 & 133.5 & 1.87 & 886 \\
\hline 0.06 & 2.74 & 1.6 & 100.8 & 1.84 & 2048 \\
\hline
\end{tabular}

the dispersion of the $\mathrm{rGO}$, such features associated with fracture can be disregarded in Fig. 7.

The images shown in Fig. $7 b, c, d, e, f$ indicate a good dispersion of $\mathrm{rGO}$ in the epoxy for the nanocomposites containing $0.01-0.05 \mathrm{wt} \%$ of rGO, and significant agglomeration could not be detected even at high magnifications. Figure $7 \mathrm{~h}$ shows a TEM image of the nanocomposite filled with $0.06 \mathrm{wt} \%$ of rGO. Isolated $\mathrm{rGO}$ sheets (indicated with black arrows) are clearly visible, which illustrate the good dispersion and relatively large flake size inherent in these nanocomposites. The infrared absorption spectra of GO, see Fig. $6 b$, show that both the surface and the edges of the GO are decorated with many OCFGs, among them epoxide groups. These OCFGs intercalated between the individual GO sheets lead to greater interlayer separation as measured for GO by XRD $(0.84 \mathrm{~nm}$, as opposed to $0.34 \mathrm{~nm}$ for GNPs, see Fig. $6 c$ ) and weaken both the van der Waals and $\pi-\pi$ interactions between sheets [11]. The overall effect is to increase compatibility between the GO and the epoxy resin, as discussed below, and increase the ease of dispersion of the GO in the epoxy.

The microstructure of the nanocomposite containing $0.06 \mathrm{wt} \% \mathrm{rGO}$ appears different to that of the lower contents, see Fig. $7 g$. Further examination at high magnifications revealed several rGO agglomerates, as shown in Fig. 8. The tendency of GO to selfassemble into liquid crystalline structures above a critical content in aqueous media, as well as in epoxy resins, has been reported [11, 16, 28-30]. Below the critical content, a more random arrangement of the GO is thermodynamically favoured, whereas at higher contents the steric hindrance between sheets and the excluded volume effect lead to self-assembly of the GO sheets into ordered structures. This may lead to agglomerated rGO nanoplatelets in the cured nanocomposites as seen in Fig. 8. This phenomenon 
may be exacerbated by a very good dispersion, as is the case in these nanocomposites, since the average statistical distance between the sheets is a minimum thereby increasing the attractive forces between them. These agglomerates could have an adverse impact on the mechanical properties of the nanocomposite, since they could act as stress contents. This is discussed in more detail in subsequent sections.

The morphology of the nanocomposites revealed a good dispersion of rGO. This has been attributed to the compatibility between the GO and the epoxy, as well as to the increased interlayer spacing between sheets due to the intercalated OCFGs which is expected to weaken the van der Waals and $\pi-\pi$ interactions between individual sheets. Although increased agglomeration of rGO was observed in the nanocomposite with $0.06 \mathrm{wt} \%$, isolated $\mathrm{rGO}$ sheets are visible which illustrate the good dispersion inherent in these nanocomposites.

\section{Thermo-mechanical properties of the nanocomposites}

The thermo-mechanical properties of the nanocomposites were examined using dynamic mechanical thermal analysis (DMTA). Figure 9a, b shows the temperature dependence of the storage modulus $\left(E^{\prime}\right)$ and the damping coefficient $(\tan \delta$ ) for each of the nanocomposites. Selected values of the storage modulus plus the glass transition temperature and height of the $\tan \delta$ peak are shown in Table 2. At $40{ }^{\circ} \mathrm{C}$, the glassy storage modulus, $E_{\mathrm{g}}{ }^{\prime}$, increases from $2.59 \mathrm{GPa}$ for the unmodified epoxy to $2.74 \mathrm{GPa}$ for the nanocomposite with $0.06 \mathrm{wt} \%$ of $\mathrm{rGO}$ (i.e. an increase of 5.8\%). In comparison, Chandrasekaran et al. [31]
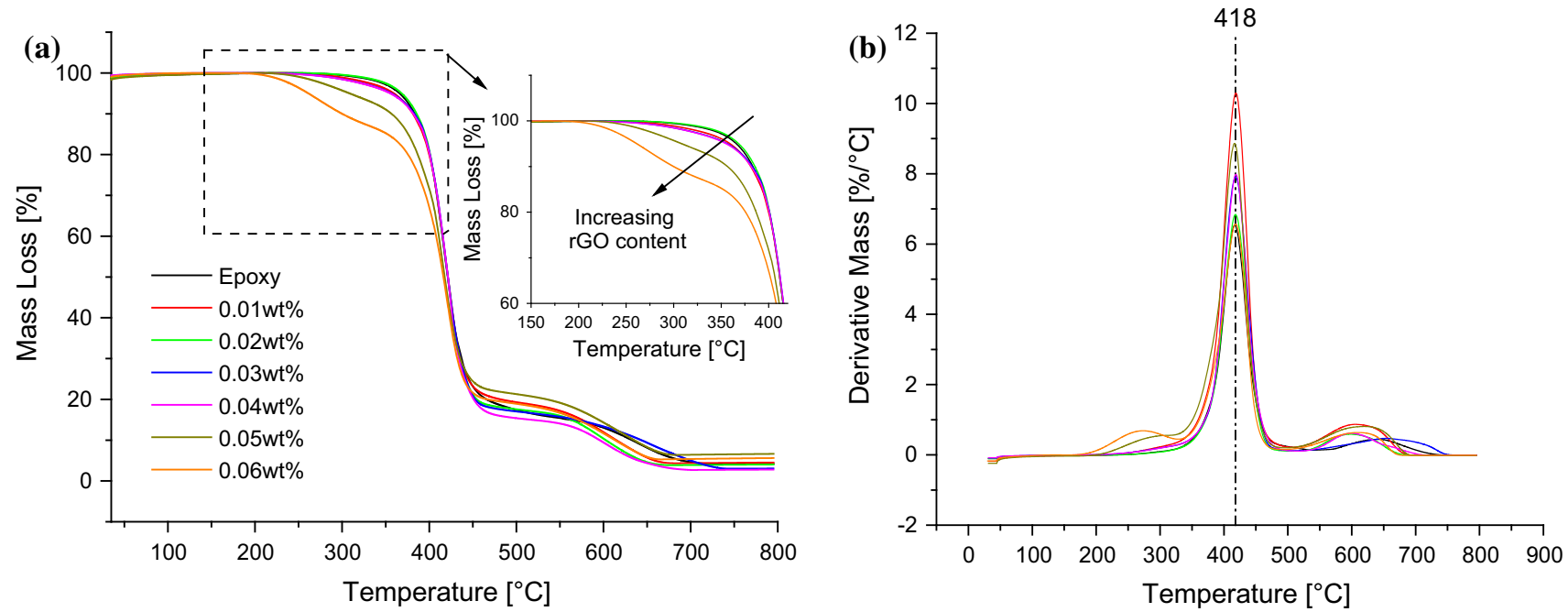

Figure 10 Thermal stability of the nanocomposites from TGA with varying rGO content, $\mathbf{a}$ mass loss and $\mathbf{b}$ derivative mass loss. The inset of a illustrates the decreasing thermal stability of the nanocomposites between 200 and $400{ }^{\circ} \mathrm{C}$ with increasing rGO content.

Table 3 Thermal stability data from TGA for the rGO/epoxy nanocomposites

\begin{tabular}{lllll}
\hline $\begin{array}{l}\text { rGO content } \\
(\mathrm{wt} \%)\end{array}$ & $\begin{array}{l}\text { Onset temperature of thermal } \\
\text { degradation }\left(T_{\text {on }}\right)\left({ }^{\circ} \mathrm{C}\right)\end{array}$ & $\begin{array}{l}\text { Mass loss at } 350{ }^{\circ} \mathrm{C} \\
\left(W_{350}\right)(\%)\end{array}$ & $\begin{array}{l}\text { Temperature of 50\% mass } \\
\text { loss }\left(T_{50 \%}\right)\left({ }^{\circ} \mathrm{C}\right)\end{array}$ & $\begin{array}{l}\text { Temperature of maximum } \\
\text { degradation }\left(T_{\max }\right)\left({ }^{\circ} \mathrm{C}\right)\end{array}$ \\
\hline 0.00 & 287.4 & 2.69 & 420.7 & 416.9 \\
0.01 & 270.6 & 3.80 & 420.7 & 418.8 \\
0.02 & 284.7 & 2.29 & 420.7 & 418.0 \\
0.03 & 257.4 & 4.36 & 421.7 & 418.3 \\
0.04 & 253.8 & 4.38 & 421.7 & 418.5 \\
0.05 & 238.9 & 8.86 & 417.3 & 416.7 \\
0.06 & 207.1 & 14.48 & 416.4 & 418.3 \\
\hline
\end{tabular}


reported an $E_{\mathrm{g}}{ }^{\prime}$ increase of only $5.1 \%$ for a GNP/ epoxy nanocomposite containing nearly double the filler content (i.e. $0.1 \mathrm{wt} \%$ of GNP), at $25^{\circ} \mathrm{C}$. The notable increase in $E_{\mathrm{g}}{ }^{\prime}$ observed in the present work is due to the high modulus of the rGO and the processing route adopted. The latter takes advantage of the relatively large flake size due to the minimal mechanical energy input during processing, the compatibility between the GO and the epoxy as well as the presence of intercalated OCFGs which lead to increased interlayer separation. These factors enable a good dispersion of the GO as seen in Fig. 7. Contributing factors to the increase in $E_{\mathrm{g}}{ }^{\prime}$, as reported in the literature, may be the wavy topology of rGO and the imperfections in the rGO lattice resulting from the $\mathrm{sp}^{3}$-hybridized domains which allow for better mechanical interlocking between the $\mathrm{rGO}$ and the polymer matrix [10]. Furthermore, the OCFGs react with the epoxy leading to the formation of covalent bonds, and therefore a better filler-matrix interface, and an increase in the load transfer characteristics is achieved, as exemplified by the observed increase in $E_{\mathrm{g}}{ }^{\prime}$ [10]. Indeed, the effective modulus of the rGO can be calculated using the rule of mixtures from the values of $E_{\mathrm{g}}{ }^{\prime}$ for the unmodified epoxy and the nanocomposite with $0.06 \mathrm{wt} \%$ of rGO. A value of 420 GPa is obtained for the effective modulus of the rGO, which compares well with literature values [32].

The glass transition temperature of the unmodified epoxy was measured to be $158.6^{\circ} \mathrm{C}$, see Table 2 . The $T_{\mathrm{g}}$ of the nanocomposites decreases with increasing rGO content, for example, the $T_{\mathrm{g}}$ decreased by $57.8{ }^{\circ} \mathrm{C}$ (i.e. $57 \%$ ) with $0.06 \mathrm{wt} \%$ of rGO, as shown in Fig. 9b. The maximum damping amplitude, as shown by the height of the tan $\delta$ peak, see Table 2, increases with increasing rGO content. These changes can generally be correlated with the crosslink density of the polymer [33]. The number average molecular weight, $M_{\text {nc }}$, between crosslinks estimated from Eq. 1 for each nanocomposite is shown in Table 2. The value of $M_{\mathrm{nc}}$ clearly increases, so the epoxy matrix becomes less highly crosslinked, with increasing rGO content. This is attributed to the fact that in situ reduction of the GO leads to the opening and/or elimination of OCFGs which may lead to the formation of covalent bonds between the GO and the epoxy matrix as mentioned previously. Furthermore, GO has been reported to promote the homopolymerization of epoxy resin [13]. These factors may change the molecular weight between crosslinks of the epoxy during crosslinking, as has been reported previously $[10,11,34,35]$. Indeed, nanoclays are known to produce a similar effect when used as fillers in epoxy polymers, e.g. [36, 37].

\section{Thermal stability of the nanocomposites}

The thermal stability of the nanocomposites in air was investigated by thermogravimetric analysis (TGA). Thermograms and derivative thermograms (DTGs) are shown in Fig. 10a, b, respectively. The thermogram of the unmodified epoxy is characterized by a two-step degradation process, see Fig. 10a. The mass loss between 350 and $450{ }^{\circ} \mathrm{C}$ can be attributed to the thermal oxidation of the epoxy, and that between 450 and $700{ }^{\circ} \mathrm{C}$ to the char formed from the thermal decomposition of the epoxy and to the rGO for the nanocomposites. Note that nothing happens below $200{ }^{\circ} \mathrm{C}$ as this was the temperature used for the thermal treatment. However, the nanocomposites are largely characterized by a threestep degradation behaviour. The extra step is the mass loss observed for the nanocomposites between 200 and $350{ }^{\circ} \mathrm{C}$ (inset of Fig. 7a), which increases with rGO content, especially at relatively high rGO contents. The mass loss observed for each of the nanocomposites up to $350{ }^{\circ} \mathrm{C}\left(W_{350}\right)$ is shown in Table 3. A steady increase in $W_{350}$ with increasing rGO content can be observed, except for the nanocomposite containing $0.02 \mathrm{wt} \% \mathrm{rGO}$. The onset temperature of thermal degradation, $T_{\text {on }}$ also decreased with increasing rGO content from $287.4{ }^{\circ} \mathrm{C}$ for the unmodified epoxy to $207.1{ }^{\circ} \mathrm{C}$ for the $0.06 \mathrm{wt} \%$ nanocomposite (i.e. a decrease of $80.3{ }^{\circ} \mathrm{C}$ ), in accordance with the trend in $T_{\mathrm{g}}$ shown in Fig. $9 \mathrm{~b}$. The inconsistencies observed in $T_{\text {on }}$ and $W_{350}$ for the 0.01 and $0.02 \mathrm{wt} \%$ rGO nanocomposites may be attributed to experimental variation. The maximum degradation temperature, $T_{\max }$ and the temperature of $50 \%$ mass loss, $T_{50 \%}$, both remained largely unchanged, see Table 3 . The changes observed in $T_{\text {on }}$ and $W_{350}$ are indicative of decreasing thermal stability with increasing rGO content, although increases in thermal stability with increasing rGO content have also been reported in the literature [11, 38].

As discussed earlier, some OCFGs remain attached to the rGO sheets after reduction, as shown in Fig. 6a, b, c, d. It therefore seems reasonable to expect an increase in the concentrations of undissociated OCFGs as the content of rGO increases. This can lead to 

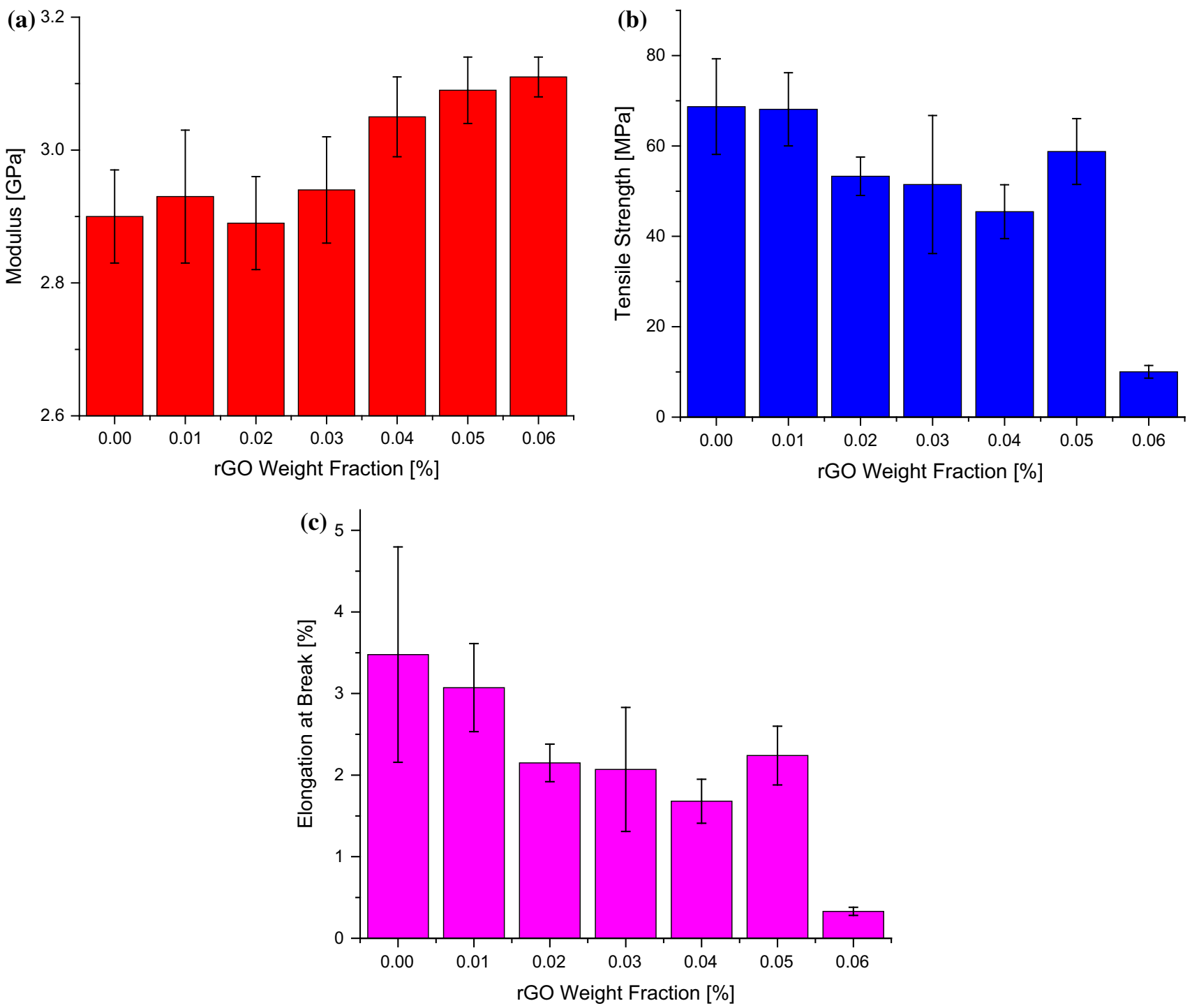

Figure 11 a Tensile modulus, $\mathbf{b}$ tensile strength and $\mathbf{c}$ elongation at break of the rGO/epoxy nanocomposites.
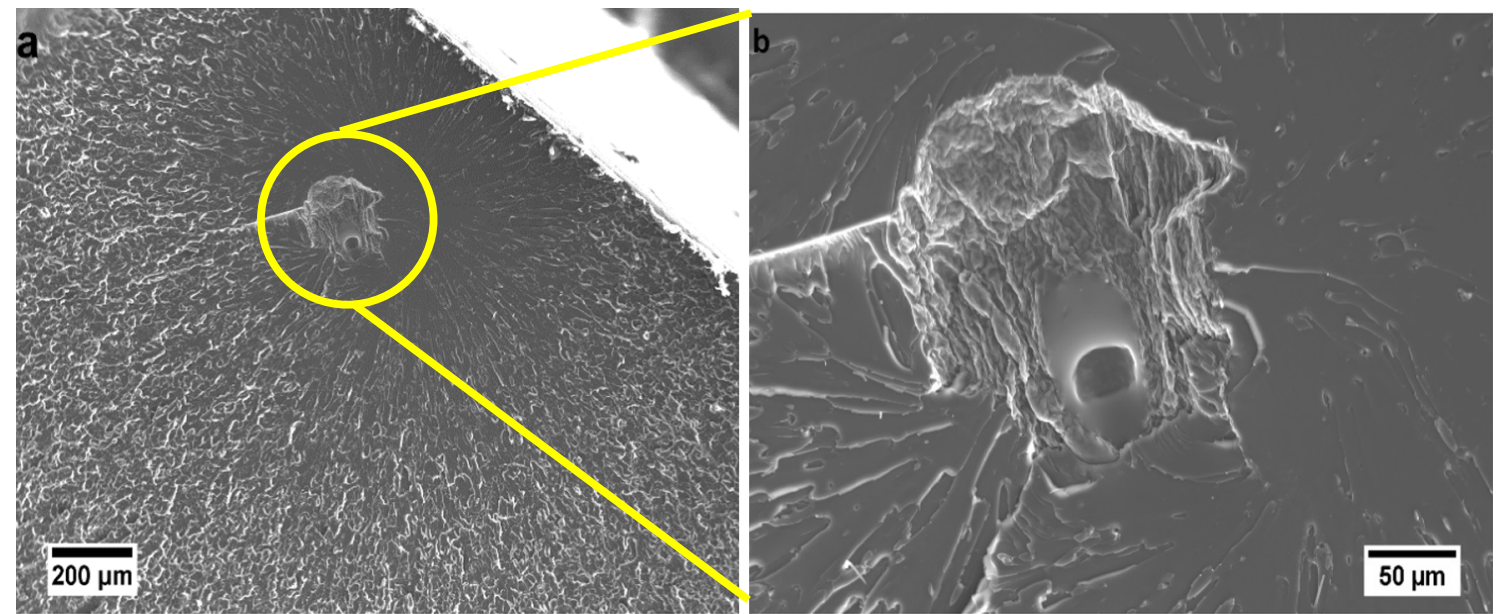

Figure $12 \mathrm{SEM}$ of the fracture surfaces of the sample containing $0.02 \mathrm{wt} \% \mathrm{rGO}$ after tensile testing, illustrating the defects present in the rGO/epoxy nanocomposites. 

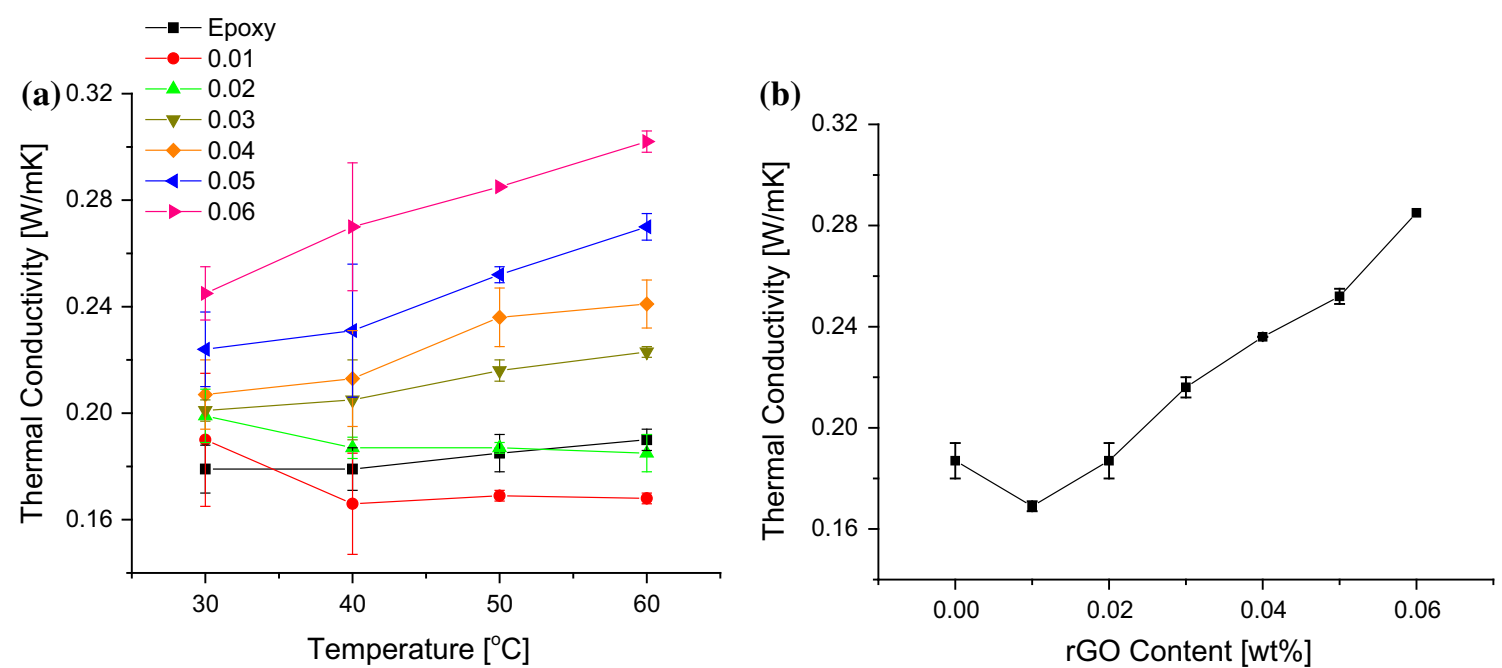

Figure 13 Thermal conductivity of unmodified epoxy and nanocomposites $\mathbf{a}$ at different temperatures and $\mathbf{b}$ at $50{ }^{\circ} \mathrm{C}$.

increasing thermal instability, as shown in Fig. 10 and Table 3, since the OCFGs may thermally dissociate at high temperatures and oxidize the epoxy matrix.

The reaction between pendant OCFGs on the GO surface and epoxy resin is well known. Yang et al. [10] have shown that DGEBA molecules can be grafted onto GO by reaction with pendant OCFGs in an aqueous medium if a mixture of GO and epoxy resin is heated at $50{ }^{\circ} \mathrm{C}$ for $4 \mathrm{~h}$. Wan et al. [39] prepared DGEBA-functionalized GO sheets by dispersing GO sheets in acetone via bath sonication in the presence of DGEBA resin at $70{ }^{\circ} \mathrm{C}$ using $\mathrm{NaOH}$ as the catalyst. They confirmed the grafting of DGEBA onto the GO sheets using a combination of XPS, XRD, AFM, TEM and Raman spectroscopy. The process adopted in this work for the removal of water $\left(60{ }^{\circ} \mathrm{C},-1000\right.$ mbar for $2 \mathrm{~h}$ ) encourages the reaction of the OCFGs on the GO with the epoxide groups in the DGEBA. Galpaya et al. [13] have shown that the OCFGs on GO can also catalyse the homopolymerization of DGEBA. As mentioned earlier, these factors have the potential to change the crosslink density and hence could be expected to affect the thermal stability of the nanocomposites.

\section{Tensile properties of the nanocomposites}

Figure 11a shows the measured tensile modulus values for the nanocomposites. Although a slight decrease in modulus for the nanocomposite with $0.02 \mathrm{wt} \%$ rGO was observed, the modulus generally increased with increasing $\mathrm{rGO}$ content from $2.90 \pm 0.07 \mathrm{GPa}$ for the unmodified epoxy to $3.11 \pm 0.03 \mathrm{GPa}$ for the nanocomposite with 0.06 wt $\%$ rGO content (i.e. 7.2\% increase). Wan et al. [39] reported a similar increase of about $7 \%$ in modulus upon the addition of $0.5 \mathrm{wt} \%$ of DGEBA-functionalized rGO to an epoxy matrix (i.e. nearly 10 times the weight of rGO used in this work), while Zaman et al. [34] observed a modest 3\% increase in modulus on adding $1 \mathrm{wt} \%$ of $4,4^{\prime}$-methylene diphenyl diisocyanate (MDI)-functionalized GNP to epoxy. The effective modulus of the rGO can be calculated from the tensile modulus values using the rule of mixtures, as was done from the values of $E_{\mathrm{g}}{ }^{\prime}$, and a value of $580 \mathrm{GPa}$ is obtained for the effective modulus of the rGO, which compares well with the 420 GPa calculated from the values of $E_{\mathrm{g}}{ }^{\prime}$ and with literature values [32]. The notable increase in modulus observed in this work may be attributed to the nanocomposite preparation route adopted, which provides for a good rGO dispersion and excellent interfacial properties between the rGO and the epoxy matrix.

The tensile strength and elongation at break of the unmodified epoxy and of the $\mathrm{rGO} /$ epoxy nanocomposites are shown in Fig. 11b, c, respectively. There is a decrease in tensile strength and elongation at break with increasing rGO content. The tensile strength decreased from $68.7 \pm 10.5 \mathrm{MPa}$ for the unmodified epoxy to $10.0 \pm 1.4 \mathrm{MPa}$ for the nanocomposite with an rGO content of $0.06 \mathrm{wt} \%$. Similarly, a decrease in the elongation at break was observed from $3.5 \pm 1.3 \%$ for the unmodified epoxy to $0.33 \pm 0.05 \%$ for the nanocomposite with $0.06 \mathrm{wt} \%$ of rGO. 
Table 4 Comparison of the thermal conductivities of graphene-based/epoxy nanocomposites at room temperature

\begin{tabular}{|c|c|c|c|c|c|c|c|c|}
\hline \# & Filler & Content & Dispersion method & $\begin{array}{l}\text { Thermal } \\
\text { conductivity } \\
(\mathrm{W} / \mathrm{mK})\end{array}$ & $\begin{array}{l}\text { Thermal } \\
\text { conductivity } \\
\text { increase (\%) }\end{array}$ & $\begin{array}{l}\text { Thermal } \\
\text { conductivity } \\
\text { enhancement } \\
\text { factor }^{\mathrm{a}}\left(\kappa_{\mathrm{e}}\right)\end{array}$ & $\begin{array}{l}\text { Measurement } \\
\text { method }\end{array}$ & Reference \\
\hline 1 & rGO & $0.06 \mathrm{wt} \%$ & Stirring & 0.25 & 38.9 & 648 & Laser flash & This work \\
\hline 2 & GNP & $20 \mathrm{wt} \%$ & Shear mixing & 5.80 & 2800 & 140 & Laser flash & {$[43]$} \\
\hline 3 & $\mathrm{MLG}^{\mathrm{b}}$ & $\begin{array}{l}10.0 \\
\text { vol \% } \\
(\sim 16.9 \\
\left.\mathrm{wt}^{\circ} \%\right)\end{array}$ & Sonication/centrifugation & 5.10 & 2300 & 136 & Laser flash & {$[44]$} \\
\hline 4 & $M_{L G}{ }^{b}$ & $\begin{array}{l}2.8 \text { vol \% } \\
(5.0 \\
\text { wt } \%)\end{array}$ & $\begin{array}{l}\text { Sonication/stirring/shear } \\
\text { mixing }\end{array}$ & 1.50 & 650 & 130 & Laser flash & {$[45]$} \\
\hline 5 & rGO & $3 \mathrm{wt} \%$ & Sonication/stirring & 1.19 & 261 & 86.9 & Hot disc & {$[46]$} \\
\hline 6 & GNP & $\begin{array}{l}5.0 \text { vol } \% \\
(\sim 8.8 \\
\left.w^{\circ} \%\right)\end{array}$ & $\begin{array}{l}\text { High shear mixing (non } \\
\text { specific) }\end{array}$ & $\sim 1.45$ & 621 & 70.6 & Laser flash & {$[47]$} \\
\hline 7 & $\begin{array}{l}\mathrm{rGO} / \text { nanosilica } \\
\text { hybrid }\end{array}$ & $1 \mathrm{wt} \%$ & Sonication/stirring & 0.32 & 61.0 & 61.0 & Laser flash & {$[48]$} \\
\hline 8 & GNP & $\begin{array}{l}2.703 \\
\text { vol } \% \\
(\sim 4.8 \\
\text { wt } \%)\end{array}$ & Sonication/stirring & $\sim 0.72$ & 243 & 50.6 & Laser flash & [49] \\
\hline 9 & $\mathrm{rGOx}^{\mathrm{c}}$ & $0.8 \mathrm{wt} \%$ & Sonication/stirring & $\sim 0.29$ & 34.0 & 42.5 & Laser flash & {$[50]$} \\
\hline 10 & rGO & $\begin{array}{l}10.0 \\
\text { vol \% } \\
(16.9 \\
\text { wt } \%)\end{array}$ & $\begin{array}{l}\text { Sonication/high shear } \\
\text { mixing }\end{array}$ & 1.26 & 556 & 32.9 & Hot disc & {$[51]$} \\
\hline 11 & rGO & $15.8 \mathrm{wt} \%$ & Sonication/stirring & 1.27 & 362 & 22.9 & Laser flash & {$[52]$} \\
\hline 12 & GNP & $3 \mathrm{wt} \%$ & Three-roll milling & $\sim 0.37$ & 68.2 & 22.7 & Laser flash & {$[53]$} \\
\hline 13 & rGO & $2 \mathrm{wt} \%$ & Three-roll milling & 0.24 & 33.3 & 16.7 & Laser flash & [14] \\
\hline 14 & GNP & $2 \mathrm{wt} \%$ & Three-roll milling & $\sim 0.21$ & 16.7 & 8.4 & Laser flash & {$[31]$} \\
\hline 15 & GNP & $\begin{array}{l}1.0 \text { vol } \% \\
(\sim 1.8 \\
\text { wt } \%)\end{array}$ & Stirring/sonication & 0.23 & 10.0 & 5.6 & Hot wire & {$[54]$} \\
\hline
\end{tabular}

a The thermal conductivity enhancement factor, $\kappa_{\mathrm{e}}$, is defined as the ratio of the percentage increase in thermal conductivity to the percentage loading by mass

b Multilayer graphene

c In situ reduced imidazole grafted GO

Examination of the fractured surfaces of the tensile samples using FEG-SEM revealed defects in the nanocomposites, as shown in Fig. 12. These defects could arise from the gases (i.e. carbon dioxide, carbon monoxide and oxygen) released during the thermal dissociation of the OCFGs as the GO is reduced. Gases such as $\mathrm{O}_{2}$ and $\mathrm{H}_{2} \mathrm{O}$, which are by-products of low-temperature reduction of the $\mathrm{GO}$, can be released during the curing of the epoxy polymer [19] and thus could be trapped in the nanocomposites as bubbles by gelation. These bubbles would tend to act as stress concentrations, thus leading to a reduction in the tensile strength of the nanocomposites as observed in Fig. 11b. Although the interaction of rGO with the matrix may have led to strong interfacial bonds between the rGO and the polymer matrix, the 
reduction in crosslink density may have made the epoxy matrix more flexible, and the presence of such defects offsets these effects. Thus, the overall effect is a reduction in the tensile strength and the elongation at break of the nanocomposites, see Fig. 11. The sharp drop observed in the tensile strength between the nanocomposite with an $\mathrm{rGO}$ content of $0.05 \mathrm{wt} \%$ $(58.8 \pm 7.3 \mathrm{MPa})$ and that of $0.06 \mathrm{wt} \%$ $(10.0 \pm 1.4 \mathrm{MPa})$, is attributed to the increased rGO agglomeration in the $0.06 \mathrm{wt} \%$ nanocomposite, as shown in Fig. 8. These observations are consistent with the sharp reduction in $T_{\mathrm{g}}$ observed from $133.5^{\circ} \mathrm{C}$ for the $0.05 \mathrm{wt} \%$ nanocomposite to $100.8^{\circ} \mathrm{C}$ for the $0.06 \mathrm{wt} \%$ nanocomposite (Fig. 9b; Table 2). Although an increase in tensile strength for an in situ chemically-reduced GO/epoxy nanocomposite has recently been reported [11], other workers [34] have noted a reduction in tensile strength in the case of MDI-functionalized GNP/epoxy nanocomposites, a phenomenon which they ascribed to the reduction in crosslink density as a result of reaction between the MDI groups and the polymer matrix. The decrease in elongation at break with increasing rGO content observed in Fig. 11c may be attributed to the strong interfacial adhesion between the rGO and the matrix.

The fracture surfaces of the tensile samples were investigated using FEG-SEM, and the resulting micrographs are shown in Fig. 7. For the unmodified epoxy, Fig. 7a shows a relatively smooth surface with river lines characteristic of a brittle fracture. An increase in the surface roughness can be observed for the nanocomposites with 0.01 and $0.02 \mathrm{wt} \%$ of rGO (see Fig. $7 b, c)$. Lines are still clearly visible on the surface, but these are not continuous, unlike for the unmodified epoxy. Instead they originate from the rGO sheets (as indicated by the arrows in Fig. 7b, c), resulting in tail-like features characteristic of crack deflection where the crack front has deviated when it encountered the particle. Such a toughening mechanism would be expected to result in enhanced fracture toughness, e.g. [40]. Chandrasekaran et al. [41] reported similar features in GNP/epoxy nanocomposites. The surface roughness increases further with increasing rGO content, as seen in Fig. $7 \mathrm{~d}-\mathrm{f}$, corresponding to nanocomposites with rGO contents of $0.03-0.05 \mathrm{wt} \%$. The agglomeration observed at 0.06 $\mathrm{wt} \%$ of rGO results in a smoother fracture surface as there are fewer points to cause crack deflection because the rGO is agglomerated into fewer thicker particles, see Fig. $7 \mathrm{~g}$.

\section{Thermal conductivity}

The thermal conductivities of the nanocomposites and the unmodified epoxy at different temperatures are shown in Fig. 13a. The thermal conductivity increases with increasing temperature and with increasing rGO content, except for the nanocomposite containing $0.01 \mathrm{wt} \%$ of $\mathrm{rGO}$. At $30{ }^{\circ} \mathrm{C}$, the thermal conductivity increases from $0.18 \pm 0.009 \mathrm{~W} / \mathrm{mK}$ for the unmodified epoxy to $0.25 \pm 0.002 \mathrm{~W} / \mathrm{mK}$ for the nanocomposite with $0.06 \mathrm{wt} \%$ of rGO (i.e. an increase of $39 \%$ ). This trend is more clearly visible in the thermal conductivity of the nanocomposites measured at $50{ }^{\circ} \mathrm{C}$, as shown in Fig. $13 \mathrm{~b}$, where an increase in thermal conductivity of $53 \%$ was measured for the $0.06 \mathrm{wt} \%$ nanocomposite $(0.29 \pm$ $0.001 \mathrm{~W} / \mathrm{mK}$ compared with $0.19 \pm 0.007 \mathrm{~W} / \mathrm{mK}$ for the unmodified epoxy). The thermal conductivities of graphene and graphene-based hybrid nanocomposites are compared in Table 4 with that from the present work. Table 4 also shows the thermal conductivity enhancement factor, $\kappa_{\mathrm{e}}$, of each of the nanocomposites, which has been defined as the increase in thermal conductivity per unit mass of filler [42]. It can be seen that the $\kappa_{\mathrm{e}}$ value of 648 is by far the highest for the nanocomposites considered in Table 4. This shows that the thermal conductivity values observed in this work are among the highest ever reported for GNP/epoxy nanocomposites with such low contents of rGO. In the authors' previous work [14], a thermal conductivity of $0.24 \mathrm{~W} / \mathrm{mK}$ was reported for a $2 \mathrm{wt} \% \mathrm{rGO} /$ epoxy nanocomposite, prepared by dispersing freeze-dried GO in epoxy via three-roll milling followed by in situ polymerization and reduction. Although this is a good increase in the thermal conductivity, the $\kappa_{\mathrm{e}}$ value of 16.7 is relatively small compared to that achieved in the present work, see Table 4 .

The thermal conductivity of particle-filled polymer composites depends on many factors: the intrinsic conductivity of filler and matrix, filler content, aspect ratio and dispersion, interfacial bonding between filler and matrix and thermal resistance offered by the interfacial layer [47]. For a highly-conductive filler such as graphene, a good dispersion will reduce the inter-particle spacing and therefore reduce the mean free path for phonon transport. This could lead to an increase in the thermal conductivity of the nanocomposite. Conversely, good interfacial adhesion between filler and matrix may form an 
interfacial layer around the filler. This will increase phonon scattering and hence reduce the thermal conductivity of the nanocomposite. At relatively low rGO contents, a good dispersion is likely to leave the rGO particles isolated, as shown in Fig. 7h. This, coupled with the interfacial layer around the rGO due to strong interfacial adhesion, will increase phonon scattering. Hence, the relatively poor thermal conductivity of the nanocomposite with $0.01 \mathrm{wt} \%$ rGO can be understood. As the content of rGO increases, the mean free path length for phonon transport decreases and the rGO particles may begin to form a network as the particles make contact with each other, as observed in the $0.06 \mathrm{wt} \% \mathrm{rGO}$ nanocomposite (Fig. 8). This may offset the phonon scattering effect due to the interfacial layer, thereby increasing the thermal conductivity of the nanocomposites as observed in the nanocomposites with rGO contents higher than $0.01 \mathrm{wt} \%$. Therefore, the excellent thermal conductivity measured in the nanocomposites can be attributed to the excellent rGO dispersion brought about by the processing technique adopted in this work.

\section{Conclusions}

In this work, a facile, scalable and commercially-viable method has been developed to prepare polymeric nanocomposites of epoxy polymer with very low rGO content, having much improved thermal conductivities. This involves taking advantage of the increased interlayer spacing in GO (compared to that in GNPs) owing to intercalated OCFGs, as well as the compatibility between the OCFGs and the matrix epoxy to achieve a good dispersion of rGO in the nanocomposite via in situ processing. The good dispersion of GO in the aqueous media was transferred to a DGEBA epoxy resin with minimal mechanical energy input. This was followed by in situ reduction of the GO to $\mathrm{rGO}$ at $200{ }^{\circ} \mathrm{C}$, which eliminated a substantial amount of the OCFGs.

Electron microscopy revealed a good dispersion of rGO in the nanocomposites, except for the highest content of $0.06 \mathrm{wt} \% \mathrm{rGO}$ where agglomeration was observed. This was attributed to the tendency of GO to form liquid crystalline structures above certain critical contents. Although addition of rGO led to a decrease in the $T_{\mathrm{g}}$ and in the resistance of the nanocomposites to thermal oxidation, the storage and tensile moduli were increased significantly. This was attributed to the excellent rGO dispersion and strong interfacial adhesion between the rGO and the epoxy matrix. The presence of defects caused by the lowtemperature reduction of the OCFGs led to a reduction in the tensile strength and elongation at break. The observed thermal conductivity of $0.25 \mathrm{~W} / \mathrm{mK}$ (measured at $30{ }^{\circ} \mathrm{C}$ for the nanocomposite with 0.06 wt $\%$ of rGO) represents an increase of $\sim 40 \%$ compared to the unmodified epoxy. This value is one of the highest thermal conductivity values ever reported for $\mathrm{rGO} /$ epoxy nanocomposites having such relatively low contents of rGO. It has been attributed to the excellent dispersion of rGO and large lateral flake size particular to these nanocomposites. These results, taken together, show that it is now possible to tune the properties of an epoxy polymer with a simple and viable method of GO addition.

\section{Acknowledgements}

This work was funded by the UK Engineering and Physical Sciences Research Council (EPSRC), Grant Number EP/K016792/1. The authors wish to thank the Department of Mechanical Engineering Sciences, University of Surrey, UK, for the X-ray photoelectron spectroscopy measurements. V.G. Rocha would like to acknowledge the European Commission (FP7 Marie Curie Intra-European Fellowships GRAPES).

Open Access This article is distributed under the terms of the Creative Commons Attribution 4.0 International License (http://creativecommons.org/ licenses/by/4.0/), which permits unrestricted use, distribution, and reproduction in any medium, provided you give appropriate credit to the original author(s) and the source, provide a link to the Creative Commons license, and indicate if changes were made.

\section{References}

[1] Kakaei K, Hasanpour K (2014) Synthesis of graphene oxide nanosheets by electrochemical exfoliation of graphite in cetyltrimethylammonium bromide and its application for oxygen reduction. J Mater Chem A 2(37):15428-15436. doi:10.1039/c4ta03026e

[2] Hummers WS, Offeman RE (1958) Preparation of graphitic oxide. J Am Chem Soc 80(6):1339 
[3] Chen T, Zeng B, Liu JL, Dong JH, Liu XQ, Wu Z, Yang XZ, Li ZM (2009) High throughput exfoliation of graphene oxide from expanded graphite with assistance of strong oxidant in modified Hummers method. J Phys Conf Ser 188:12051. doi:10.1088/1742-6596/188/1/012051

[4] Marcano DC, Kosynkin DV, Berlin JM, Sinitskii A, Sun Z, Slesarev A, Alemany LB, Lu W, Tour JM (2010) Improved synthesis of graphene oxide. ACS Nano 4(8):4806-4814. doi:10.1021/nn1006368

[5] Pei S, Cheng H (2012) The reduction of graphene oxide. Carbon 50(9):3210-3228. doi:10.1016/j.carbon.2011.11.010

[6] Zhang C, Lv W, Zhang W, Zheng X, Wu M, Wei W, Tao Y, Li Z, Yang Q (2014) Reduction of graphene oxide by hydrogen sulfide: a promising strategy for pollutant control and as an electrode for Li-S batteries. Adv Energy Mater 4(7):1301565. doi:10.1002/aenm.201301565

[7] Ai K, Liu Y, Lu L, Cheng X, Huo L (2011) A novel strategy for making soluble reduced graphene oxide sheets cheaply by adopting an endogenous reducing agent. J Mater Chem 21(10):3365-3370. doi:10.1039/c0jm02865g

[8] Huang X, Qi X, Boey F, Zhang H (2012) Graphene-based composites. Chem Soc Rev 41(2):666-686. doi:10.1039/ c1cs $15078 \mathrm{~b}$

[9] Kuilla T, Bhadra S, Yao D, Kim NH, Bose S, Lee JH (2010) Recent advances in graphene based polymer composites. Prog Polym Sci 35(11):1350-1375. doi:10.1016/j.progpo lymsci.2010.07.00

[10] Yang H, Shan C, Li F, Zhang Q, Han D, Niu L (2009) Convenient preparation of tunably loaded chemically converted graphene oxide/epoxy resin nanocomposites from graphene oxide sheets through two-phase extraction. J Mater Chem 19(46):8856-8860. doi:10.1039/b915228h

[11] Yousefi N, Lin X, Zheng Q, Shen X, Pothnis JR, Jia J, Zussman E, Kim J (2013) Simultaneous in situ reduction, self-alignment and covalent bonding in graphene oxide/ epoxy composites. Carbon 59:406-417. doi:10.1016/j.car bon.2013.03.034

[12] Tang H, Ehlert GJ, Lin Y, Sodano HA (2012) Highly efficient synthesis of graphene nanocomposites. Nano Lett 12(1):84-90. doi:10.1021/nl203023k

[13] Galpaya DG, Fernando JF, Rintoul L, Motta N, Waclawik ER, Yan C, George GA (2015) The effect of graphene oxide and its oxidized debris on the cure chemistry and interphase structure of epoxy nanocomposites. Polymer 71:122-134. doi:10.1016/j.polymer.2015.06.054

[14] Olowojoba GB, Eslava S, Gutierrez ES, Kinloch AJ, Mattevi C, Rocha VG, Taylor AC (2016) In situ thermally reduced graphene oxide/epoxy composites: thermal and mechanical properties. Appl Nanosci 6:1015-1022. doi:10.1007/s13204016-0518-y
[15] Peng M, Tang X, Zhou Y (2016) Fast phase transfer of graphene oxide from water to triglycidyl para-aminophenol for epoxy composites with superior nanosheet dispersion. Polymer 93:1-8. doi:10.1016/j.polymer.2016.03.016

[16] Aboutalebi SH, Gudarzi MM, Zheng QB, Kim J (2011) Spontaneous formation of liquid crystals in ultralarge graphene oxide dispersions. Adv Funct Mater 21(15):2978-2988. doi:10.1002/adfm.201100448

[17] Li M, Gao C, Hu H, Zhao Z (2013) Electrical conductivity of thermally reduced graphene oxide/polymer composites with a segregated structure. Carbon 65:371-373. doi:10.1016/j. carbon.2013.08.016

[18] Pearson RA, Yee AF (1989) Toughening mechanisms in elastomer-modified epoxies. Part 3. The effect of cross-link density. J Mater Sci 24:2571-2580. doi:10.1007/BF01174528

[19] Zhou S, Bongiorno A (2013) Origin of the chemical and kinetic stability of graphene oxide. Sci Rep 3:2484. doi:10. 1038/srep02484

[20] Jeong H, Lee YP, Jin MH, Kim ES, Bae JJ, Lee YH (2009) Thermal stability of graphite oxide. Chem Phys Lett 470(4-6):255-258. doi:10.1016/j.cplett.2009.01.050

[21] Acik M, Lee G, Mattevi C, Chhowalla M, Cho K, Chabal YJ (2010) Unusual infrared-absorption mechanism in thermally reduced graphene oxide. Nat Mater 9(10):840-845. doi:10. 1038/nmat2858

[22] Acik M, Mattevi C, Gong C, Lee G, Cho K, Chhowalla M, Chabal YJ (2010) The role of intercalated water in multilayered graphene oxide. ACS Nano 4(10):5861-5868. doi:10.1021/nn101844t

[23] Bagri A, Mattevi C, Acik M, Chabal YJ, Chhowalla M, Shenoy VB (2010) Structural evolution during the reduction of chemically derived graphene oxide. Nat Chem 2(7):581-587. doi:10.1038/nchem.686

[24] Kim H, Miura Y, Macosko CW (2010) Graphene/polyurethane nanocomposites for improved gas barrier and electrical conductivity. Chem Mater 22(11):3441-3450. doi: $10.1021 / \mathrm{cm} 100477 \mathrm{v}$

[25] Fu C, Zhao G, Zhang H, Li S (2013) Evaluation and characterization of reduced graphene oxide nanosheets as anode materials for lithium-ion batteries. Int $\mathrm{J}$ Electrochem Sci 8:6269-6280

[26] Ju HM, Choi SH, Huh SH (2010) X-ray diffraction patterns of thermally-reduced graphenes. J Korean Phys Soc 57(61):1649-1652

[27] Hull D (1999) Fractography: observing, measuring, and interpreting fracture surface topography. Cambridge University Press, Cambridge

[28] Xu Z, Gao C (2011) Graphene chiral liquid crystals and macroscopic assembled fibres. Nat Commun 2:571. doi:10. 1038/ncomms 1583 
[29] Park JH, Aluru NR (2011) Self-assembly of graphenes. Surf Sci 605(17-18):1616-1620. doi:10.1016/j.susc.2011.02.011

[30] Konatham D, Striolo A (2008) Molecular design of stable graphene nanosheets dispersions. Nano Lett 8(12):4630-4641. doi:10.1021/n1802262p

[31] Chandrasekaran S, Seidel C, Schulte K (2013) Preparation and characterization of graphite nano-platelet (GNP)/epoxy nano-composite: mechanical, electrical and thermal properties. Eur Polym J 49(12):3878-3888. doi:10.1016/j.eur polymj.2013.10.008

[32] Suk JW, Piner RD, An J, Ruoff RS (2010) Mechanical properties of monolayer graphene oxide. ACS Nano 4(11):6557-6564. doi:10.1021/nn101781v

[33] Nielsen LE, Landel RF (1994) Mechanical properties of polymers and composites, 2nd edn. M. Dekker, New York

[34] Zaman I, Phan TT, Kuan H, Meng Q, La LTB, Luong L, Youssf O, Ma J (2011) Epoxy/graphene platelets nanocomposites with two levels of interface strength. Polymer 52(7):1603-1611. doi:10.1016/j.polymer.2011.02.003

[35] Ma J, Meng Q, Michelmore A, Kawashima N, Izzuddin Z, Bengtsson C, Kuan H (2013) Covalently bonded interfaces for polymer/graphene composites. J Mater Chem A 1(13):4255-4264. doi:10.1039/c3ta01277h

[36] Liu W, Hoa SV, Pugh M (2005) Organoclay-modified high performance epoxy nanocomposites. Compos Sci Technol 65(2):307-316. doi:10.1016/j.compscitech.2004.07.012

[37] Marouf BT, Bagheri R, Pearson RA (2008) Observation of two $\alpha$-relaxation peaks in a nanoclay-filled epoxy compound. J Mater Sci 43(21):6992-6997. doi:10.1007/s10853008-3023-1

[38] Qiu SL (2011) Effects of graphene oxides on the cure behaviors of a tetrafunctional epoxy resin. Express Polym Lett 5(9):809-818. doi:10.3144/expresspolymlett.2011.79

[39] Wan Y, Tang L, Gong L, Yan D, Li Y, Wu L, Jiang J, Lai G (2014) Grafting of epoxy chains onto graphene oxide for epoxy composites with improved mechanical and thermal properties. Carbon 69:467-480. doi:10.1016/j.carbon.2013. 12.050

[40] Kinloch AJ, Taylor AC (2006) The mechanical properties and fracture behaviour of epoxy-inorganic micro- and nanocomposites. J Mater Sci 41(11):3271-3297. doi:10.1007/ s10853-005-5472-0

[41] Chandrasekaran S, Sato N, Tölle F, Mülhaupt R, Fiedler B, Schulte K (2014) Fracture toughness and failure mechanism of graphene based epoxy composites. Compos Sci Technol 97:90-99. doi:10.1016/j.compscitech.2014.03.014
[42] Zhao Y, Zhang Y, Bai S (2016) High thermal conductivity of flexible polymer composites due to synergistic effect of multilayer graphene flakes and graphene foam. Compos Part A Appl S 85:148-155. doi:10.1016/j.compositesa.2016.03. 021

[43] Ganguli S, Roy AK, Anderson DP (2008) Improved thermal conductivity for chemically functionalized exfoliated graphite/epoxy composites. Carbon 46(5):806-817. doi:10. 1016/j.carbon.2008.02.008

[44] Shahil KMF, Balandin AA (2012) Graphene-multilayer graphene nanocomposites as highly efficient thermal interface materials. Nano Lett 12(2):861-867. doi:10.1021/ nl203906r

[45] Shen X, Wang Z, Wu Y, Liu X, He Y, Kim J (2016) Multilayer graphene enables higher efficiency in improving thermal conductivities of graphene/epoxy composites. Nano Lett 16(6):3585-3593. doi:10.1021/acs.nanolett.6b00722

[46] Xie F, Qi SH, Wu D (2016) A facile strategy for the reduction of graphene oxide and its effect on thermal conductivity of epoxy based composites. Express Polym Lett 10(6):470-478. doi:10.3144/expresspolymlett.2016.45

[47] Yu A, Ramesh P, Itkis ME, Bekyarova E, Haddon RC (2007) Graphite nanoplatelet-epoxy composite thermal interface materials. J Phys Chem C 111(21):7565-7569. doi:10.1021/ jp071761s

[48] Hsiao M, Ma CM, Chiang J, Ho K, Chou T, Xie X, Tsai C, Chang L, Hsieh C (2013) Thermally conductive and electrically insulating epoxy nanocomposites with thermally reduced graphene oxide-silica hybrid nanosheets. Nanoscale 5(13):5863-5871. doi:10.1039/c3nr01471a

[49] Min C, Yu D, Cao J, Wang G, Feng L (2013) A graphite nanoplatelet/epoxy composite with high dielectric constant and high thermal conductivity. Carbon 55:116-125. doi:10. 1016/j.carbon.2012.12.017

[50] Lei L, Shan J, Hu J, Liu X, Zhao J, Tong Z (2016) Co-curing effect of imidazole grafting graphene oxide synthesized by one-pot method to reinforce epoxy nanocomposites. Compos Sci Technol 128:161-168. doi:10.1016/j.compscitech.2016. 03.029

[51] Liu C, Hu G (2015) Highly efficient reduction of graphene oxide by sub/supercritical water and their application for thermal interface materials. Appl Therm Eng 90:193-198. doi:10.1016/j.applthermaleng.2015.07.011

[52] Han D, Zhao Y, Zhang Y, Bai S (2015) Vertically and compactly rolled-up reduced graphene oxide film/epoxy composites: a two-stage reduction method for graphene- 
based thermal interfacial materials. RSC Adv 5(114):9442694435. doi:10.1039/c5ra16780a

[53] Wang F, Drzal LT, Qin Y, Huang Z (2015) Mechanical properties and thermal conductivity of graphene nanoplatelet/epoxy composites. J Mater Sci 50(3):1082-1093. doi:10. 1007/s10853-014-8665-6
[54] Hung MT, Choi O, Ju YS, Hahn HT (2006) Heat conduction in graphite-nanoplatelet-reinforced polymer nanocomposites. Appl Phys Lett 89(2):23117. doi:10.1063/1.2221874 